\title{
Respiratory Rhythm Entrainment by Somatic Afferent Stimulation
}

\author{
Jeffrey T. Potts, ${ }^{1}$ Ilya A. Rybak, ${ }^{2}$ and Julian F. R. Paton ${ }^{3}$ \\ ${ }^{1}$ Department of Physiology, Wayne State University School of Medicine, Detroit, Michigan 48201, ${ }^{2}$ School of Biomedical Engineering, Science and Health \\ Systems, Drexel University, Philadelphia, Pennsylvania 19104, and 32Department of Physiology, School of Medical Sciences, University of Bristol, Bristol BS8 \\ 1TD, United Kingdom
}

Respiratory and locomotor patterns are coupled during locomotion. The objectives of this study were to (1) demonstrate that respiratory rhythms are entrained by sensory input from somatic afferents, (2) establish whether the parabrachial nucleus mediates entrainment, (3) examine responses of single respiratory neurons in the ventral respiratory group (VRG) to somatic afferent stimulation, and (4) use a computational model of the pontomedullary respiratory network (Rybak et al., 2004a,b) to suggest neuronal mechanisms for entrainment.

We used an in situ preparation in young rats that retained pontomedullary respiratory circuits and spinal pathways transmitting somatosensory input. We demonstrate that rhythmic stimulation of somatic afferents entrains respiratory rhythm on a 1:1 basis (1:1), increasing breathing frequency up to $\sim 1.4-2.2$ times greater than spontaneous frequency. Stable entrainment occurred only when stimuli were delivered during expiration. Reversible blockade of the lateral parabrachial nucleus eliminated entrainment. Somatic afferent stimulation produced significant increases in the firing rate of augmenting expiratory (E2) neurons but shortened the firing duration of postinspiratory (post-I) neurons. A computational model reproduced 1:1 entrainment and other experimental findings based on the assumption that the somatic afferents initiate early onset of inspiration via activation of medullary E2 neurons. The model also predicted that afferent stimulation evoked transient hyperpolarization of ramp-inspiratory (ramp-I) neurons. This was confirmed experimentally by intracellular recording from ramp-I neurons. Our experimental and modeling results demonstrate that an entrainment pathway from somatic afferents to the VRG via the lateral parabrachial nucleus causes resetting of respiratory rhythm through excitation of $\mathrm{E} 2$ and consequent inhibition of post-I neurons.

Key words: respiratory control; somatosensory input; pontomedullary respiratory network; entrainment; sensory-motor integration; locomotion

\section{Introduction}

Entrainment of respiratory and locomotor rhythms has been well documented in mammals, including the rat, cat, dog, horse, humans, and birds (Krogh and Lindhard, 1913; Dejours, 1967; Bramble and Carrier, 1983; Funk et al., 1992; Iscoe, 1981; Ainsworth et al., 1996). Coupling of these rhythms occurs over a wide frequency range $(1: 1,2: 1,3: 2$, and $5: 3)$, and this coordination is thought to be produced by feedforward mechanisms (Zunzt and Geppert, 1886; Johansson, 1893; Krogh and Lindhard, 1913; Eldridge et al., 1981), spinal feedback mechanisms (Kao, 1963; Iscoe and Polosa, 1976; Brice et al., 1988; Brown et al., 1988; Fernandes et al., 1990; Pan et al., 1990), or the activity of spinal locomotor pattern generators (Dubayle and Viala, 1996, 1998). The central neural mechanisms that mediate the coordination

Received Sept. 24, 2004; revised Jan. 7, 2005; accepted Jan. 8, 2005.

This work was supported by National Institutes of Health Grants HL059167 (J.T.P.), NS046062-2 (I.A.R.), and HL072415-01 (I.A.R.), Children's Research Center of Michigan and Children's Hospital of Michigan Grant CRCM440017 (J.T.P.), and British Heart Foundation Grant BS93003 (J.F.R.P.)

Correspondence should be addressed to Dr. Jeffrey T. Potts, Veterinary Biomedical Science, Dalton Cardiovascular Research Center, University of Missouri, 134 Research Park Drive, Columbia, M0 65211. E-mail: pottsj@@missouri.edu.

DOI:10.1523/JNEUROSCI.3881-04.2005

Copyright $\odot 2005$ Society for Neuroscience $\quad$ 0270-6474/05/251965-14\$15.00/0 between respiratory and locomotor pattern generators are poorly understood. Recently, Morin and Viala (2002) demonstrated that sensory input from the lumbar spinal cord in the en bloc neonatal rat preparation in vitro entrained respiratory rhythm and evoked GABA-mediated IPSPs in phrenic motoneurons (PMNs). They proposed that IPSPs may contribute to entrainment by modulating the excitability of PMNs to coincide with descending command signals from the medullary respiratory network. Importantly, they showed that entrainment required an intact brainstem. However, the nature of the brainstem connections mediating entrainment, as well as the effect of sensory input on medullary respiratory neurons, has not been investigated.

The goals of the present study were as follows: (1) demonstrate that respiratory rhythms can be entrained by sensory input from somatic afferents using a reduced in situ preparation of young rats that preserves spinal sensory pathways and pontomedullary respiratory circuits; (2) establish whether the parabrachial nucleus mediates the effects of somatic afferents on the respiratory rhythm, because this region is known to play a crucial role in controlling the timing of respiratory phase switching (Lumsden, 1923; Cohen, 1971; Cohen and Feldman, 1977; Wang et al., 1993; Dick et al., 1994; St. John, 1998); and (3) examine the effect of somatic afferent stimulation on respiratory neurons in 
the ventral respiratory group (VRG) complex because this region is crucial for respiratory rhythmogenesis (Cohen, 1979; Feldman, 1986; Richter et al., 1986; von Euler, 1986; Bianchi et al., 1995; Richter, 1996). Finally, we used a recently developed computational model of the pontomedullary respiratory network (Rybak et al., 2004a,b) to investigate the possible neural mechanisms responsible for the respiratory rhythm entrainment by somatic afferent activation.

Parts of this work have been published previously in abstract form (Potts and Paton, 2000, 2001, 2002).

\section{Materials and Methods}

\section{In situ working heart-brainstem preparation}

Experiments were performed on juvenile male rats (6-8 weeks of age; 65-90 g; Sprague Dawley). We recently modified the working heartbrainstem preparation (WHBP) (Paton, 1996a) to facilitate activation of somatosensory afferents, which included those innervating skeletal muscle (Potts et al., 2000). This preparation produces a spontaneous, eupneic-like pattern of phrenic discharge and exhibits many characteristics of respiratory function, including control of inspiratory offswitching by vagus nerve stimulation (to mimic the Hering-Breuer reflex) and switching to apneustic and gasping-like patterns after lesions to the pons (Paton, 1996b; St. John and Paton, 2000; St. John et al., 2002). The surgical preparation and extracorporeal circuit have been described in detail previously (Paton, 1996a,b; Potts et al., 2000). Briefly, animals were deeply anesthetized with halothane $(3-5 \%)$ via spontaneous inhalation and transected subdiaphragmatically. The depth of anesthesia was assessed by the absence of a corneal reflex and lack of limb withdrawal to a noxious pinch. The upper body (head, forelimbs, and thorax) was submerged immediately in ice-cold Ringer's solution bubbled with a $95 \% \mathrm{O}_{2}-5 \% \mathrm{CO}_{2}$ gas mixture. A precollicular decerebration was performed to remove all influences from rostral brain regions, and a portion of the posterior thorax was removed to expose the heart and lungs. The thoracic aorta and the left phrenic nerve (PN) were isolated by blunt dissection. After surgery, preparations were transferred to an acrylic chamber in which the thoracic aorta was cannulated in the retrograde direction with a double-lumen catheter (16 and 18 ga; Braintree Scientific, Braintree, MA) with the aid of a binocular microscope (Stemi SV11; Zeiss, Oberkochen, Germany). The tip of the catheter was advanced to the aortic arch under direct visual inspection. Perfusion of Ringer's solution gassed with a $95 \% \mathrm{O}_{2}-5 \% \mathrm{CO}_{2}$ gas mixture was commenced immediately using a digital pump equipped with a multi-roller pump head (model 505S pump; model 314DW2 pump head; Watson-Marlow, Wilmington, MA). The perfusate was warmed to $32-34^{\circ} \mathrm{C}$ and passed through two in-line bubble traps and a filter (polypropylene mesh; pore size, $40 \mu \mathrm{m}$ ). Oncotic pressure of the perfusate was $\sim 310 \mathrm{mOsm}$ per kilogram of $\mathrm{H}_{2} \mathrm{O}$. Pump flow rate was monitored via an in-line volumetric flow probe (model 2N; Transonic Systems, Ithaca, NY), and perfusion pressure was measured via a strain-gauge pressure transducer (model P23; Gulton Statham, Costa Mesa, CA). At the flow rates used in the present study $(30-40 \mathrm{ml} / \mathrm{min})$, systemic perfusion pressure averaged $70-90 \mathrm{mmHg}$. The electrocardiogram (EKG) was recorded by means of fine silver wires placed directly on the heart. The EKG waveform was amplified (model P511; Astro-Med, West Warwick, RI), filtered (100 Hz to $3 \mathrm{kHz}$ ), and displayed on an oscilloscope. A discriminator circuit (NeuroLog NL 201; Digitimer, Hertfordshire, UK) was used to generate transistor-transistor logic (TTL) pulses triggered from the upstroke of $\mathrm{R}$-wave. Instantaneous heart rate was derived from the measured interbeat interval and displayed in beats per minute (bpm). Neuromuscular paralysis was produced by addition of vecuronium bromide $(50 \mu \mathrm{g})$ directly to the perfusate. Elapsed time from the start of anesthesia to the initiation of systemic perfusion was $10 \mathrm{~min}$.

\section{Activation of somatic afferents}

Somatic afferents innervating the forelimb were activated by direct electrical stimulation of forelimb flexors (biceps brachii and coracobrachialis muscle groups). The ipsilateral scapula was stabilized using a modified vertebral clamp, the distal end of the forelimb was attached to a forcedisplacement transducer (FT-100; CB Science), and developed tension was measured. Stimuli were delivered via two fine silver wires $(0.005 \mathrm{inch}$ outer diameter; A-M Systems, Carlsborg, WA) introduced into the forelimb flexor groups with the aid of a stainless steel trocar (20 ga). Each wire was inserted $\sim 10 \mathrm{~mm}$ parallel to the humerus. The average distance between these wires was $8 \mathrm{~mm}$. Train pulse stimuli $(0.18-1.0 \mathrm{~Hz} ; 50-100$ $\mathrm{ms}$ train duration; $1-3 \mathrm{~ms}$ pulse duration; $250 \mu \mathrm{A}$ to $1 \mathrm{~mA}$ ) generated by a stimulator (model S88; Astro-Med) via a stimulation isolation unit (model PSIU6; Astro-Med) were used. This stimulus was sufficient to activate afferents as evidenced by the evoked tachypnea and pressor/ tachycardic responses.

\section{Microinjection of $G A B A_{A}$ receptor agonist isoguvacine in parabrachial nucleus}

Four barrels of a glass multibarrel micropipette (10-15 $\mu \mathrm{m}$ outer diameter) were filled with isoguvacine $(3 \mathrm{~mm})$, pontamine sky blue $(2 \%)$, glutamate (10 mM), and Ringer's solution (vehicle). The multibarrel pipette was mounted in a three-dimensional micromanipulator (Soma Scientific Instruments, Irvine, CA) and advanced into the pons using a micro-stepper motor (model 6000 ULN; Burleigh Instruments, Fishers, NY). Microinjections were performed using a picopump (model PV820; World Precision Instruments, Sarasota, FL), and the microinjected volumes were directly measured by observing the movement of the liquid meniscus using a stereomicroscope fitted with a calibrated eyepiece reticule. All volume injections were $<50 \mathrm{nl}$. Injection sites were based on previously published data (Chamberlin and Saper, 1994) and were functionally identified as sites that increased the frequency and/or amplitude of the phrenic nerve discharge (PND) (i.e., hyperpneic response) after chemical stimulation by glutamate.

\section{Neural activity recording}

Whole-nerve activity was recorded from the PN and the central vagus nerve $(\mathrm{CVN})$ using glass suction electrodes (tip diameter, $0.2-0.3 \mathrm{~mm}$ ). The raw neurograms were amplified (NeuroLog NL104; Digitimer), integrated (NeuroLog NL703; Digitimer) using a time constant of $100 \mathrm{ms,}$ filtered (NeuroLog NL126; $500 \mathrm{~Hz}$ to $5 \mathrm{kHz}$; Digitimer), and displayed on an analog oscilloscope (model 7603; Tektronix, Wilsonville, OR).

Extracellular recording was performed using single-barrel glass microelectrodes $(1.5 \mathrm{~mm}$ outer diameter, $0.84 \mathrm{~mm}$ inner diameter; World Precision Instruments) with tip diameters of 1-2 $\mu \mathrm{m}$ outer diameter using a vertical pipette puller (model 700C; David Kopf Instruments, Tujunga, CA) and filled with $3 \mathrm{M} \mathrm{NaCl}$. Impedance was $15-30 \mathrm{M} \Omega$ as measured in saline. The recording electrode was mounted on a three-axis micromanipulator (Somapatch, model MM-1R; Soma Scientific Instruments). The VRG complex was stereotaxically mapped using a grid matrix $(100 \times 100 \mu \mathrm{m})$ measured from calamus scriptoris (CS). Successful recordings from respiratory neurons were obtained between 400-600 $\mu \mathrm{m}$ rostral to CS and $1400-1800 \mu \mathrm{m}$ lateral to the midline. The electrode was advanced in the $z$-axis $(1800-2500 \mu \mathrm{m})$ using a piezoelectric stepper motor (model IW-711-01; Burleigh Instruments) driven by an ultralow-noise controller (model 6200ULN-1-1; Burleigh Instruments). The electrical activity from single-unit VRG neurons was amplified by an AC amplifier (2000-5000×; NeuroLog NL104; Digitimer) and filtered (500 $\mathrm{Hz}$ to $3 \mathrm{kHz}$; NeuroLog NL126; Digitimer). Action potential trajectories were monitored on-line using a window discriminator (NeuroLog NL201; Digitimer) and a storage oscilloscope (40 mHz, model 55140; Kikisui). Unit discrimination was performed in real time with dualthreshold windowing to capture both the trajectory and amplitude of each action potential. Neuronal activity was recorded to a computer using 16-bit analog-to-digital conversion (micro 1401; Cambridge Electronics Design, Cambridge, UK) and commercially available software (Spike 2, version 4.17; Cambridge Electronics Design). Wave-shape template-matching algorithms (Spike2; Cambridge Electronics Design) were used off-line to confirm the wave shape of individual action potentials.

Intracellular recordings of inspiratory VRG neurons were recorded using sharp glass microelectrodes (borosilicate glass, GC150F; Clark Electromedical Instruments, Reading, UK) pulled on a Sutter Instru- 
ments (Novato, CA) P-87 micropipette puller (Brown-Flaming). Pipettes were filled with $3 \mathrm{M} \mathrm{KCl}$ (DC resistance, 60-120 M $\Omega$ ). Membrane potentials were recorded using an intracellular amplifier (Axoclamp 2A; Axon Instruments, Union City, CA) in bridge mode. VRG neurons were impaled by passing a transient $(<2 \mathrm{~ms})$ high-amplitude positive current pulse. Only inspiratory neurons with a membrane potential of less than $-50 \mathrm{mV}$ (measured during the expiratory period) were included in these analyses.

\section{Experimental protocol}

Frequency dependence $(\mathrm{n}=14$ rats). The frequency of somatic stimulation was first chosen so that it evoked entrainment at a constant latency. We defined entrainment as the phasic discharge of PN activity during somatic afferent stimulation that occurred at constant latency. This was achieved by calculating a poststimulus timed histogram (PSTH) between the contraction-evoked increase in forelimb tension and the onset of the subsequent PN discharge over a 30 s period. Forelimb contraction was initially performed at $110 \%$ of the spontaneous rate of PN discharge. Thereafter, stimulation frequency was incrementally increased until the cycle-to-cycle latency became variable and entrainment no longer occurred in a 1:1 ratio. Data obtained from this series of experiments was used to construct a frequency-response profile for respiratory entrainment by somatic afferent input

Phase dependence $(\mathrm{n}=11$ rats). Rectifying and integrating the PN signal in real time and generating a TTL-timing pulse from the upstroke of the rectified-integrated phrenic neurogram was used to trigger somatic afferent stimulation in discrete phases of the respiratory cycle. A delay $(10-100 \mathrm{~ms})$ was applied to the timing pulse to trigger the stimulator and contract the forelimb. A custom-written script (developed in our laboratory with assistance from Cambridge Electronics Design) was used to perform these operations. Forelimb twitch was evoked within discrete phases of the respiratory cycle, and the latency of the evoked PN response $\left(l_{\mathrm{s}}\right)$ was measured. In addition, these data were used to construct a phase-response plot. For this analysis, the phase of stimulus $\left(\phi_{\mathrm{s}}\right)$ was calculated as the time interval from the onset of integrated PN burst to the stimulus application $\left(t_{\mathrm{s}}\right)$ divided by the mean respiratory cycle duration $\left(P_{\mathrm{m}}\right)$ defined as the time between onsets of two sequential integrated PN bursts averaged over the three respiratory cycles preceding stimulus application. The phase shift $(\Delta \phi)$ was calculated as the difference between the durations of the perturbed $\left(P_{\mathrm{s}}\right)$ and unperturbed $\left(P_{\mathrm{m}}\right)$ cycles normalized to $P_{\mathrm{m}}$.

Role of parabrachial nucleus ( $\mathrm{n}=6$ rats). Somatic afferents were stimulated at a frequency that represented $110 \%$ of spontaneous PN discharge rate for $30 \mathrm{~s}$. Isoguvacine was then microinjected to hyperpneic sites within the lateral parabrachial nucleus, and somatic afferents were restimulated at the initial stimulation frequency. Contraction was than repeated at 2, 5, 10, and $20 \mathrm{~min}$ after injection to assess recovery. A previous study showed that washout and recovery of isoguvacine occurred within 15-20 min (Boscan and Paton, 2002). During recovery, an equivalent volume of Ringer's solution was microinjected into the same site (volume control), and somatic stimulation was repeated. This site was then marked by microinjection of pontamine sky blue. A maximum of one site per side per animal was used to facilitate accurate mapping of the lateral parabrachial nucleus.

Peripheral nerve recording and defining the respiratory phase durations. The effect of somatic afferent input on inspiratory (I) and expiratory (E) durations was determined using CVN and PN recordings. CVN and PN activities were recorded before and during somatic afferent stimulation. From these recordings, I phase duration was determined from the PN burst, and E duration was determined as the time between the end of the PN burst and the onset of the subsequent burst. The duration of the respiratory cycle was therefore equal to the sum of I and E durations. The border between postinspiration (E1 phase of expiration) and late expiration (E2 phase) was defined using CVN recording, by assuming that the end of postinspiratory phase corresponded to the time of abrupt reduction of postinspiratory activity observed in this nerve (see Fig. 6A). Hence, the post-I duration was defined as the time from the end of inspiration (determined from the phrenic neurogram) to the termina- tion of post-I activity estimated from CVN activity. The duration of E2 phase was then calculated as $\mathrm{E}$ duration minus post-I duration.

Extracellular recording ( $\mathrm{n}=12$ rats). The effect of somatic afferent input on the discharge activity of single-unit expiratory neurons in the VRG complex was determined. Recordings were obtained from post-I and E2 neurons based on their discharge patterns with respect to PN and CVN activity. Forelimb twitch triggered from the phrenic burst was evoked within discrete phases of the respiratory cycle (see above, Experimental protocol, Phase dependence) and the neuronal responses of expiratory VRG neurons to a train of somatic stimuli (50-100 ms train duration; $1-3 \mathrm{~ms}$ pulse duration; $250 \mu \mathrm{A}$ to $1 \mathrm{~mA}$ ) were obtained. Somatic-evoked changes in the firing frequency and duration of VRG neurons were then measured and quantified.

Intracellular recording ( $\mathrm{n}=8$ rats). Finally, after successful impalement and bridge balancing, the responses of postinspiratory and rampinspiratory neurons in the VRG complex to somatic afferent stimulation were recorded intracellularly in a separate group of animals. In most experiments, when somatic activation evoked hyperpolarization of membrane potential neurons were injected with negative current $(-0.5$ to $-2 \mathrm{nA}$ ) to reduce membrane potential below the chloride equilibrium potential (approximately $-70 \mathrm{mV}$ ). To facilitate stable intracellular recording of membrane potential, the contracting forelimb was stabilized by a modified vertebral clamp that was attached to a magnetic base and fastened to the medial border of the scapula.

\section{Solutions}

The Ringer's solution contained the following (in mM): $125 \mathrm{NaCl}, 24$ $\mathrm{NaHCO}_{3}, 5 \mathrm{KCl}, 2.5 \mathrm{CaCl}_{2}, 1.25 \mathrm{MgSO}_{4}, 1.25 \mathrm{KH}_{2} \mathrm{PO}_{4}, 10 \mathrm{D}$-glucose, and an oncotic agent (Ficoll, $70 \mathrm{kDa}, 1.25 \%$; Sigma, St. Louis, MO). Vecuronium bromide ( $0.04 \mu \mathrm{g} / \mathrm{ml}$, Norcuron; Organon, West Orange, NJ) was added to the perfusate to block neuromuscular transmission.

\section{Statistical analyses}

All data were collected on-line using commercially available data acquisition hardware and software (micro1401 analog-to-digital converter, Spike 2 software, version 4.15; Cambridge Electronics Design). Signals were analyzed using custom-designed script files. PSTHs were performed using Spike 2 software to examine the latency of contraction-evoked PN responses. The effect of stimulus frequency on phase-resetting variables, phrenic burst latency and properties of intracellularly recorded neurons were analyzed using one-way ANOVA (SAS Institute, Cary, NC). When a statistical significant main effect was found, differences between means were analyzed by post hoc (Student-Newman-Keuls) analyses. Data are presented as mean \pm SEM. For all analysis, differences were considered significant if $p<0.05$.

\section{Computational modeling}

To investigate possible neural mechanisms responsible for the respiratory rhythm entrainment by somatic afferent input, we used a computational model of the pontomedullary respiratory network. Construction and validation of this model has been described previously in detail by Rybak et al. (2004a,b). Here we provide a short description of the model. The model contains interacting populations of respiratory neurons that were characterized in the rostroventrolateral medulla and pons in vivo. The medullary component of the model includes three major regions: rostroventral respiratory group (rVRG), pre-Bötzinger complex (pre$\mathrm{BötC}$ ), and Bötzinger complex (BötC). The pontine component is conditionally subdivided into a rostral (rPons) and caudal (cPons) parts. The neural populations of the rPons in the model are considered to perform the functions of the nucleus parabrachialis medialis, Kölliker-Fuse nucleus, and other respiratory areas located in the dorsolateral and ventrolateral pons. The following neural populations are included in the model: ramp-I and late-I (both in rVRG); early-I and pre-I (in pre-BötC); post-I and E2 (in BötC); I-modulated, E-modulated, and IE-modulated (in rPons); and tonic (in cPons). All neurons were modeled in the Hodgkin-Huxley style and incorporated biophysical properties and channel kinetics characterized in respiratory neurons in vitro. Specifically, the fast sodium current and the persistent sodium current (included only in pre-I neurons) were described using recent experimental data obtained from the studies of acutely dissociated neurons 
obtained from the pre-BötC of the rat (Rybak et al., 2003a). The high-threshold and lowthreshold calcium currents (not present in pre-I neurons) were described using data of Elsen and Ramirez (1998). Intracellular calcium dynamics was based on data of Frermann et al. (1999). Other cellular parameters were accepted from the previous models (Rybak et al., 1997a-c, 2003b). Each population consisted of 50 neurons. Neuronal parameters (the reversal potential for leakage current defining neuronal excitability), weights of synaptic connections, and initial conditions for all variables were randomized over each population. Synaptic transmission was modeled to meet characteristics of fast glutamatergic, glycinergic, and GABAergic synapses with parameters adjusted to reproduce the shape of the corresponding synaptic potentials observed in intracellular recordings from VRG neurons (Rybak et al., 1997b, 2003b, 2004c). Connectivities among the medullary neural populations were assigned using the existing direct and indirect physiological data. Some unknown connections, such as inhibitory connections from the post-I to the pre-I population, were included to support the inspiratory off-switch (IOS) and expiratory off-switch (EOS) mechanisms suggested (for details, see Rybak et al., 2004a,b). The network interactions among rVRG and BötC populations (ramp-I, early-I, and late-I) and between these populations and BötC populations define the basic circuitry for IOS mechanism. The late-I population plays the key role in the initiation of inspiratory offswitching (Cohen, 1979; Ogilvie et al., 1986; Cohen et al., 1993; Richter, 1996; Rybak et al., 1997b) by providing inhibition of the early-I population. The latter disinhibits the post-I population that completes switching to expiration and makes it irreversible. Interactions among BötC populations (post-I and E2) and between post-I and pre-I populations of preBötC define the basic circuitry for the EOS mechanism, with the pre-I population explicitly performing the inspiratory on-switching (and hence the EOS) function (Richter, 1996). During expiration, the pre-I population is inhibited by the post-I population. When the pre-I population fires, after a release from inhibition, it provides the initial activation to the early-I and ramp-I population, which complete switching to inspiration. Reciprocal excitatory connections are assigned between the medullary ramp-I and the pontine I and IE populations, and between the medullary post-I and the pontine IE and E populations. These connections provide I, IE, or E modulation of activity of the corresponding pontine populations. The model suggests the existence of topically organized bidirectional mapping between the BötC-VRG areas in the medulla and the corresponding respiration-related areas in the rostral pons (Gaytán et al., 1997). This model has been shown capable of reproducing many respiratory phenomena, including the Hering-Breuer reflex, effects of vagotomy, apneusis produced by elimination of the rostral pons, as well as the transition from eupnea to
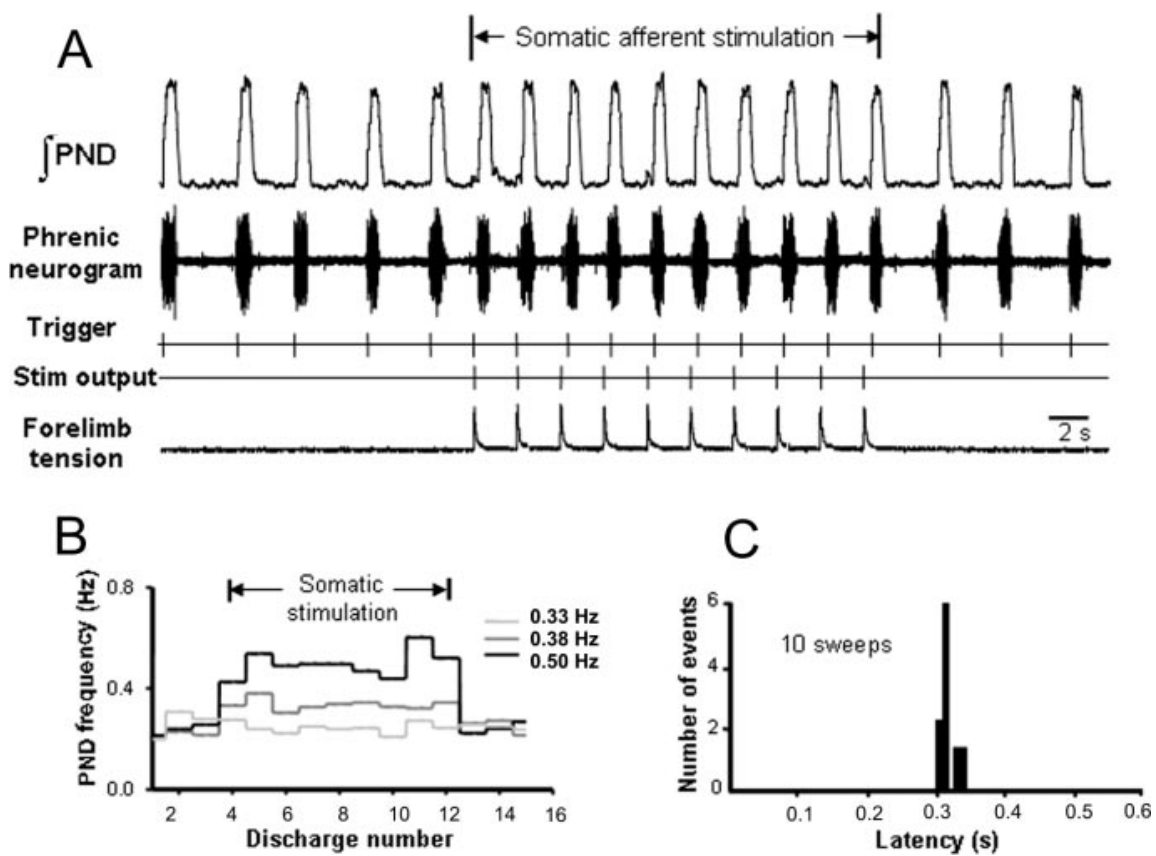

Figure 1. Modulation and entrainment of respiratory-locomotor rhythms by somatic afferent activation in the WHBP. A, PND is phase coupled to forelimb contraction. $\boldsymbol{B}$, Entrainment (1:1) evoked over a range of stimulation frequencies. $\boldsymbol{C}$, Poststimulus timed histogram showing nearly constant latency of evoked phrenic nerve activity during somatic stimulation.

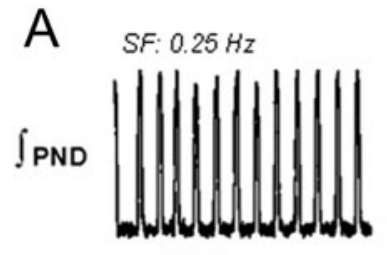

Tension
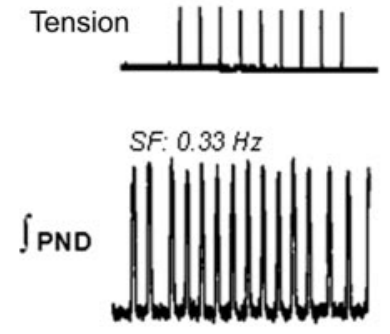

Tension
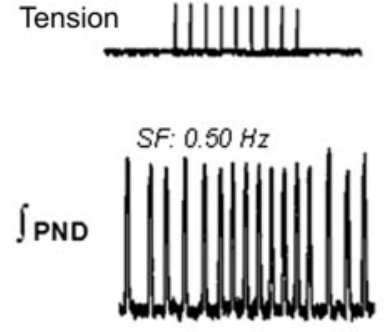

Tension $\quad\|\|\|\|$ is
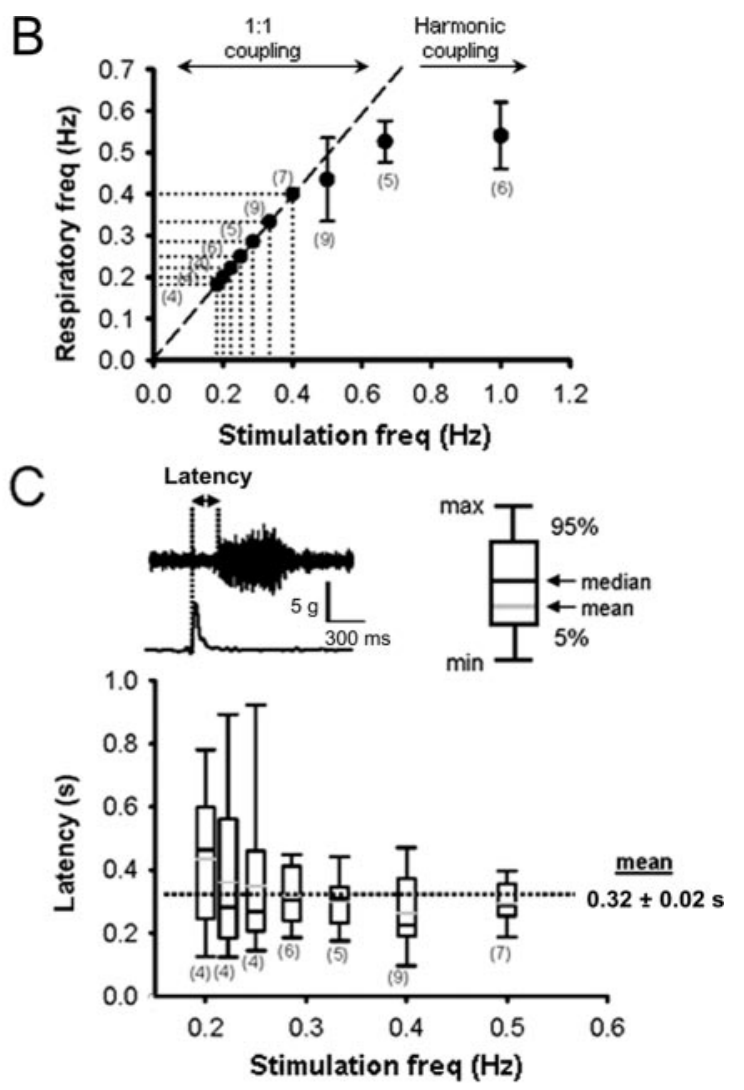

Figure 2. Respiratory rhythm entrainment (1:1) by activation of somatic afferent fibers. $\boldsymbol{A}$, Recordings of integrated PND and forelimb tension over a range of stimulation frequencies (SF). $\boldsymbol{B}$, Scatter plot showing relationship between respiratory and somatic stimulation frequencies. Numbers in parentheses indicate the number of animals. Line of identity indicates 1:1 coupling. Note that, when somatic stimulation exceeded $0.5 \mathrm{~Hz}$, coupling occurred at harmonic frequencies and 2:1 coupling was produced at $1 \mathrm{~Hz}$. For analysis of data not conforming to 1:1 coupling, see Figure 3. C, Box plot showing latency of the phrenic burst relative to stimulation frequency. Note that this analysis was performed only on data conforming to 1:1 coupling. 

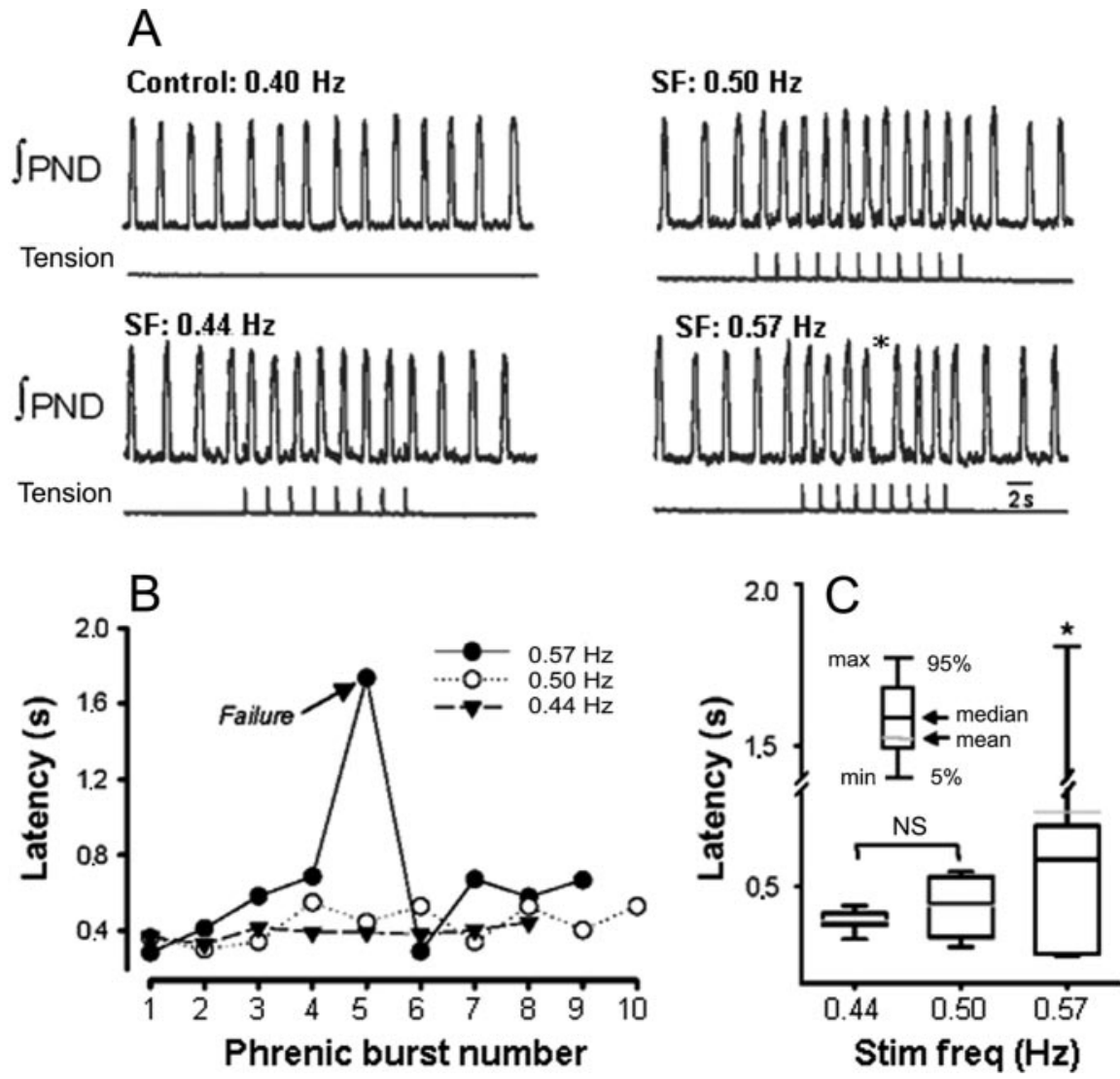

Figure 3. Example of harmonic coupling of respiratory and somatic rhythms. $A$, Recordings of integrated PND and forelimb tension at increasing stimulation frequency (SF). Note that somatic stimulation at $0.57 \mathrm{~Hz}$ was associated with a "dropped" phrenic burst $\left(^{*}\right) . \boldsymbol{B}$, Latency of the phrenic burst measured during somatic stimulation. Note that latency progressively lengthened with increasing somatic stimulation frequency. $C$, Box plot of latency of the phrenic burst and stimulation frequency (Stim freq). Note that somatic stimulation at $0.57 \mathrm{~Hz}$ was associated with a significant increase in latency. ${ }^{*} p<0.05$, significantly different from 0.44 and $0.50 \mathrm{~Hz}$.

gasping-like pattern after complete removal of the pons (Rybak et al., 2004a,b). To fit the experimental conditions of this study (i.e., absence of lung inflation, and thus, no Hering-Breuer reflex), the vagal feedback loop in the model was eliminated.

To simulate the effects of somatic afferent stimulation on the respiratory network performance and the respiratory rhythm generated, an additional neural population (S) has been incorporated in the model, specifically in the rostral pons (conditionally in the area of lateral parabrachial nucleus). Based on the experimental data present here, we have suggested that this pontine population receives an excitatory input from somatic afferents and transmits this excitation to the medullary E2 population. The square wave pulses (50-200 $\mathrm{ms}$ in duration) were applied to the $\mathrm{S}$ pontine population to imitate stimulation of somatic afferents.

All simulations were performed on a Pentium IV computer $(2.6 \mathrm{GHz}$, 512 MB; Dell Computer Company, Round Rock, TX), with a Windows XP operating system using a simulation package NSM 2.0, developed at Drexel University (by I. A. Rybak, S. N. Markin, D. G. Ivashko, and N. A. Shevtsova) using Microsoft (Seattle, WA) Visual C ++ . Differential equations were solved using the exponential von Euler integration method with a step of $0.1 \mathrm{~ms}$ (for details, see Rybak et al., 2003b, 2004c).

\section{Results}

Frequency dependence of entrainment by somatic afferent stimulation ( $n=14$ rats)

The in situ WHBP generated a spontaneous pattern of respiratory motor activity characterized by rhythmic, ramp-like PN discharges with frequency ranging from 0.18 to $0.4 \mathrm{~Hz}$ (period, 2.5$5.5 \mathrm{~s}$ ) and inspiratory duration of $490 \pm 30 \mathrm{~ms}$. The average systemic perfusion pressure was $75 \pm 8$ $\mathrm{mmHg}$, and average heart rate was $375 \pm$ $22 \mathrm{bpm}$. We arbitrarily separated our data into two groups for analysis: (1) somatic afferent-evoked 1:1 entrainment; and (2) somatic afferent-evoked entrainment at different coupling ratios.

A representative example of 1:1 entrainment is shown in Figure 1. Entrainment was characterized by a rapid increase in respiratory frequency that was phase locked to somatic stimulation. The onset latency between somatic stimuli and resultant PN bursts was determined using a poststimulus timed histogram. In the example shown in Figure 1, endogenous PN frequency was $0.33 \mathrm{~Hz}$, and somatic stimulation at 0.38 and $0.50 \mathrm{~Hz}$ successfully entrained (1:1) phrenic respiratory bursts without failures (Fig. $1 B$ ). The average onset latency measured over 10 consecutive contraction cycles was $332 \mathrm{~ms}$ (Fig. 1C).

Forelimb contraction was performed over a range of stimulus frequencies. For all experiments $(n=14$ rats $)$, the frequency range for $1: 1$ entrainment varied from 0.2 to $0.7 \mathrm{~Hz}$. A representative example is shown in Figure $2 A$. In this example, the spontaneous breathing frequency was $0.18 \mathrm{~Hz}$. When the frequency of somatic stimulation was in the range of $0.18-0.4$ $\mathrm{Hz}$, the phrenic bursts were entrained (1: $1)$. Figure $2 B$ shows that $1: 1$ phase coupling was not maintained when stimulation frequency exceeded $0.4 \mathrm{~Hz}$. Instead, the onset of phrenic bursts failed to coincide with the stimulus, and entrainment occurred at harmonic ratios (i.e., 2:1). The onset latency of PN bursting (i.e., the delay between stimulus application and evoked PN burst) during 1:1 entrainment remained nearly constant over stimulus frequencies $(320 \pm 20 \mathrm{~ms})$, although its variability (Max - Min) tended to increase at low frequencies (Fig. 2C).

An example of the lack of 1:1 entrainment during somatic afferent stimulation is illustrated in Figure $3 A$. In this experiment, respiratory rhythm (with spontaneous frequency of 0.4 $\mathrm{Hz}$ ) was entrained by somatic stimulation at 0.44 and $0.5 \mathrm{~Hz}$. (Fig. $3 B$ ). However, when stimulus frequency was increased to $0.57 \mathrm{~Hz}, 1: 1$ entrainment failed (Fig. 3A, asterisk, "dropping" of a PN burst). At this stimulation frequency, onset latency progressively increased over the first four stimulation cycles until entrainment failed on the fifth stimulus. Group data of the median onset latencies are shown in Figure 3C. Latency at 0.44 and 0.50 Hz was $392 \pm 12$ and $439 \pm 27 \mathrm{~ms}$, respectively $(n=14 ; p=\mathrm{NS})$. However, onset latency was significantly increased at $0.57 \mathrm{~Hz}$ $(658 \pm 144 \mathrm{~ms} ; p<0.05)$. Likewise, the range of onset latency (Max latency - Min latency) was significantly greater at $0.57 \mathrm{~Hz}$ when compared with 0.50 and $0.44 \mathrm{~Hz}(1450 \pm 150$ vs $114 \pm 10$ and $249 \pm 22 \mathrm{~ms} ; p<0.05)$. Together, these data suggest that there is an "optimal range" over which somatic afferent stimulation can entrain (1:1) respiration. It is only this 1:1 entrainment that we have studied further. 

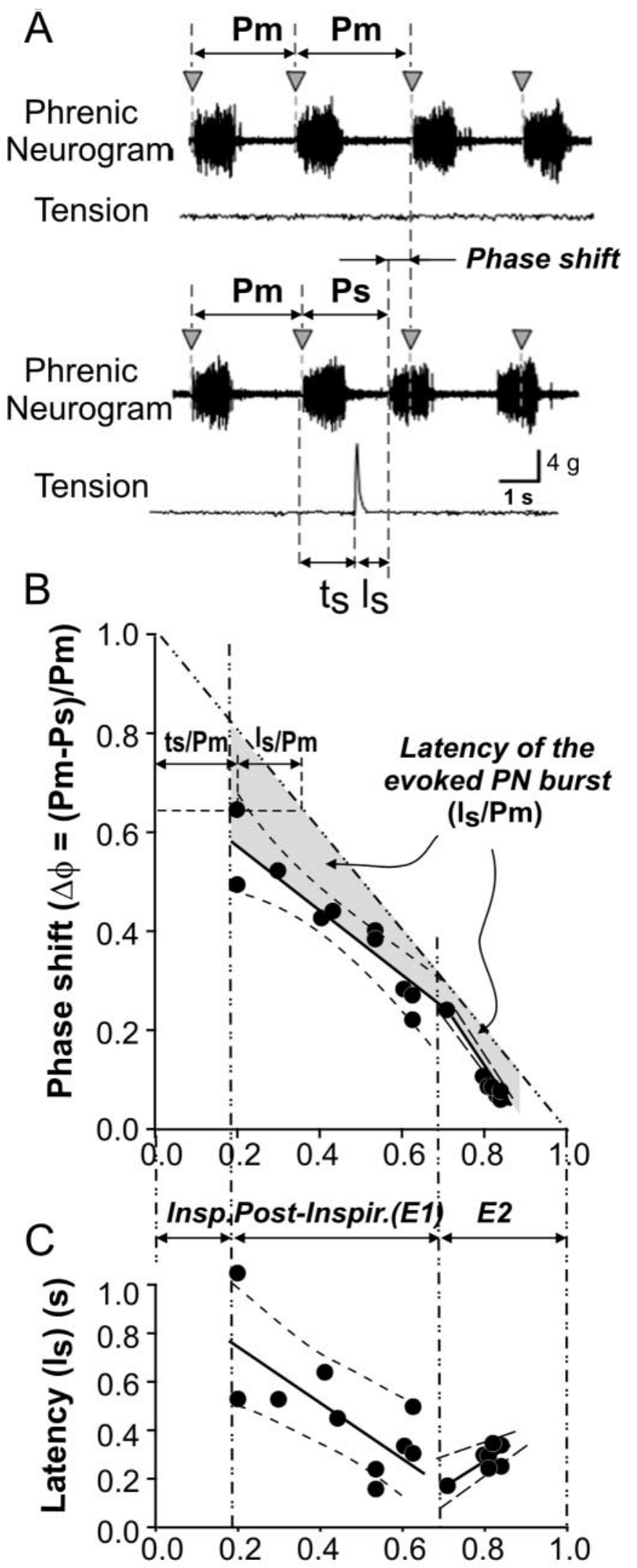

\section{Stimulus phase $\left(\phi_{\mathrm{s}}=\mathrm{t}_{\mathrm{s}} / \mathrm{Pm}\right)$}

Figure 4. Phase resetting of spontaneous respiratory rhythms by somatic stimulation. $\boldsymbol{A}$, Continuous record showing the effect of stimulation-evoked muscle contraction on spontaneous phrenic nerve activity. Vertical arrowheads represent expected occurrence of spontaneous non-evoked phrenic burst activity. Note that somatic stimulation during expiration produced
Entrainment (1:1) is respiratory phase dependent $(n=11$ rats $)$

To determine the phase dependence of the effect of somatic afferent stimulation on resetting of respiratory rhythms, stimuli were delivered randomly at discrete phases during expiration. Stimuli were applied during postinspiratory (post-I or E1 phase of stimulus; $\left.\phi_{\mathrm{s}} \approx 0.2-0.7\right)$ and late-expiratory $\left(\mathrm{E} 2 ; \phi_{\mathrm{s}} \approx 0.7-1.0\right.$ ) phases of expiration. An example of data obtained from one experiment is shown in Figure 4. Each stimulus caused a premature onset of the following phrenic burst and a corresponding phase shift in the phrenic pattern (Fig. 4A). Respiratory rhythm resetting was consistently produced when the stimulus occurred during the expiration (Fig. $4 B)$. The phase shift $(\Delta \phi)$ consisted of two components: the phase of stimulus arrival or stimulus phase $\left(\phi_{\mathrm{s}}=t_{\mathrm{s}} / P_{\mathrm{m}}\right)$, and the latency in the resulting phase resetting $\left(l_{\mathrm{s}}\right)$ normalized to the average unperturbed phase duration $\left(P_{\mathrm{m}}\right)$ : $\Delta \phi=\left(P_{\mathrm{m}}-P_{\mathrm{s}}\right) / P_{\mathrm{m}}=1-\left(\phi_{\mathrm{s}}+l_{\mathrm{s}} / P_{\mathrm{m}}\right)$. The phase shift negatively correlated with the stimulus phase during both expiratory phases (post-I phase, slope of $-0.70, r^{2}=0.85$; E2 phase, slope of $-1.26, r^{2}=0.98$ ) (Fig. $4 B$ ). The latency of phase resetting was estimated separately for the post-I and E2 phases. During the post-I phase, the latency progressively decreased (slope of -1.06), whereas during the E2 phase, it tended to increase (slope of 0.93 ) (Fig. 4C). However, despite shortening of expiration (because of the earlier onset of the next PN burst), there was no significant difference in the amplitude or duration between the spontaneous and stimulus-evoked PN bursts (duration of spontaneous PN burst was $708 \pm 34$ vs $666 \pm 36 \mathrm{~ms}$ for the evoked burst; $F$ value $=0.7 ; p>0.05$ ). In contrast, when the stimulus coincided during inspiration, the pattern of evoked PN activity was inconsistent. Depending on stimulus intensity and phase of stimulus application during inspiration, the PN burst could be transiently inhibited with or without shortening of the reevoked inspiratory burst but without an effect on the duration of subsequent expiration (data not shown). Together, these results demonstrate that (1) rhythm resetting of endogenous respiratory network activity by somatic afferent stimulation is phase dependent, and (2) stable phase resetting is restricted to timing of the stimulus application during expiration.

\section{Role of parabrachial nucleus in somatic afferent-evoked respiratory entrainment ( $n=6$ rats)}

An example of neuronal inactivation by isoguvacine microinjection into a glutamate-sensitive site on respiratory entrainment is shown in Figure 5. Before isoguvacine, somatic afferent stimulation entrained respiratory rhythm and increased average PND

$\longleftarrow$

premature bursting of the phrenic nerve. $\boldsymbol{B}$, Phase-response plot depicting phase shift of stimulus-evoked phrenic burst relative to the stimulus phase. The vertical dash-dot lines separate inspiratory, postinspiratory, and E2 phases. Phase resetting was estimated separately for postinspiratory and E2 phases. The solid line in the area corresponding to each phase is a linear fit of the data with $99 \%$ confidence limits (dashed lines). For the postinspiratory phase, this line had slope of $-0.70, r^{2}=0.85$; for $E 2$ phase, slope of $-1.26, r^{2}=0.97$. The gray-filled area represents latency of the evoked PND bursts normalized to prestimulus respiratory duration $\left(I_{\mathrm{s}} / P_{\mathrm{m}}\right)$. For explanation and description of performed calculations, see Materials and Methods. $\boldsymbol{C}$, Absolute latency of evoked PND as a function of phase of stimulus application. Similar to $\boldsymbol{B}$, the latency was estimated separately for the postinspiratory (Post-Inspir.) and E2 phases. The solid lines in the postinspiratory and E2 areas represent linear fit of the data with $99 \%$ confidence limits (dashed lines). For the postinspiratory phase, slope of $-1.06 \mathrm{~s}, r^{2}=0.52$; for $E 2$ phase, slope of $0.93 \mathrm{~s}, r^{2}=0.67$. As seen in $\boldsymbol{B}$ and $\boldsymbol{C}$, the latency of PND bursts decreased with increasing stimulus phase during the post-I phase and increased during the E2 phase. Insp., Inspiratory. 
A
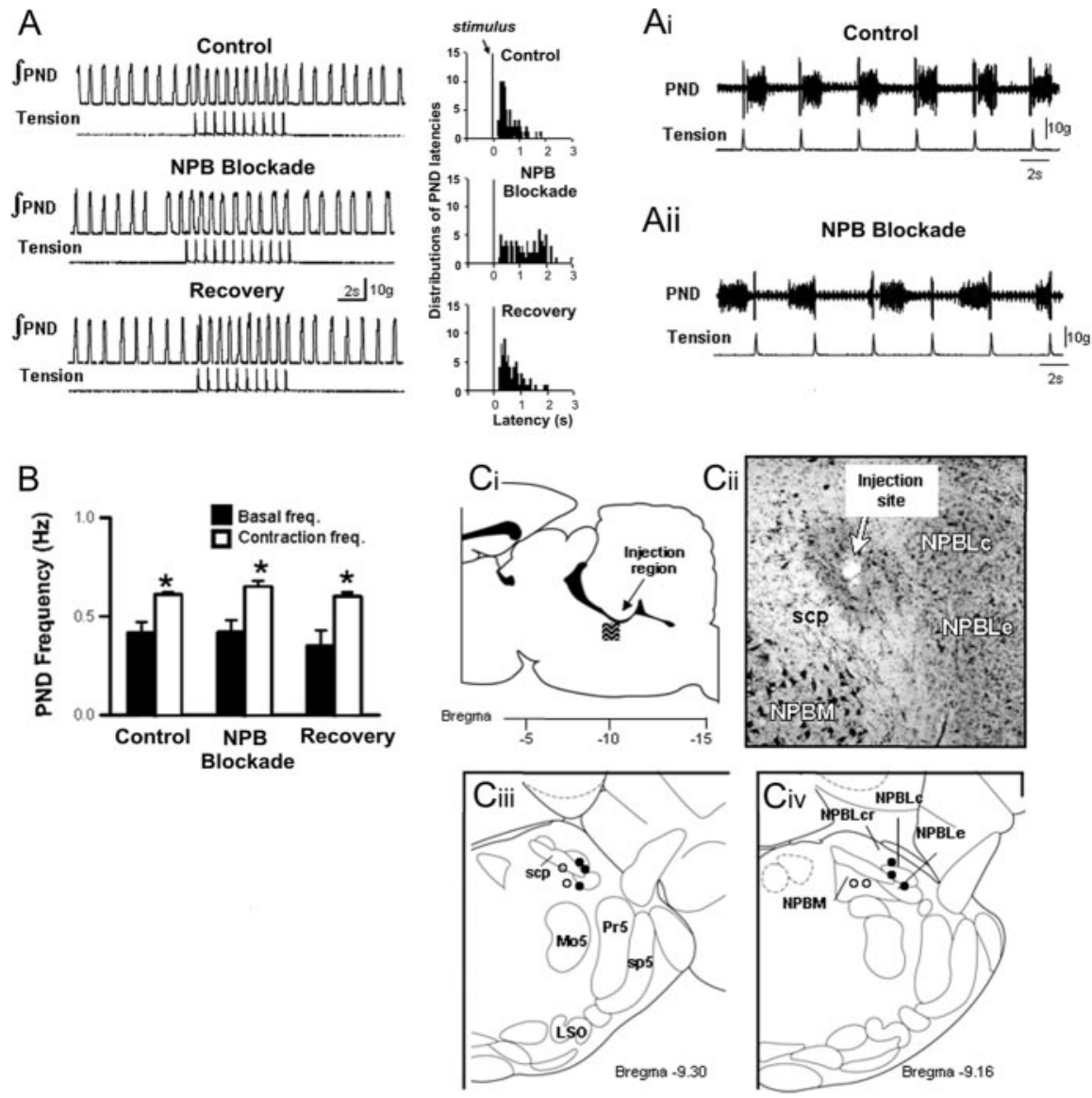

Figure 5. Effect of blockade of the parabrachial nucleus (NPB) on respiratory-somatic entrainment. $\boldsymbol{A}$, Recordings of integrated PND and stimulus-evoked forelimb tension before isoguvacine microinjection (Control), immediately after unilateral injection into the parabrachial nucleus (NPB Blockade) and after washout (Recovery). Right panels illustrate poststimulus timed histogram of the latency of evoked PND and somatic stimulation. Ai and Aii show that the consistent relationship between somatic stimulation and the onset of PND ( $\boldsymbol{A} \boldsymbol{i}$ ) is disrupted after parabrachial nucleus blockade (Aii). $\boldsymbol{B}$, Effect of synaptic blockade of the parabrachial nucleus on tachypnea evoked by muscle contraction. Note that, although entrainment is disrupted by isoguvacine, the increase in PND frequency was preserved. ${ }^{*} p<0.05$, significantly different from basal frequency. Ci-Civ, Summary of unilateral injections of isoguvacine into the parabrachial nucleus. Filled circles illustrate injection sites that blocked entrainment. Note that these injections were clustered in the lateral parabrachial nucleus (NPBL). Open circles illustrate injection sites that failed to block entrainment. Note that these sites were generally medial to the superior cerebellar peduncle (scp). KF, Kölliker-Fuse nucleus; Mo5, motor trigeminal nucleus; Pr5, principal 5 nucleus; sp5, spinal trigeminal tract; LSO, lateral superior olive; NPBLe, lateral parabrachial nucleus, external; NPBLc, lateral parabrachial nucleus, central; NPBLcr, lateral parabrachial nucleus, crescent; NPBM, medial parabrachial nucleus.

frequency by $38 \%(0.45 \pm 0.04$ vs $0.60 \pm 0.04 \mathrm{~Hz}$, control vs stimulus; $p<0.05)$. The PSTH showed that PND occurred at constant onset latency ( $350 \pm 52 \mathrm{~ms})$ (Fig. 5Ai). Unilateral microinjection of isoguvacine into the region of the lateral parabrachial nucleus failed to alter the basal respiratory frequency $(0.45 \pm 0.04$ vs $0.40 \pm 0.03 \mathrm{~Hz}$, control vs isoguvacine; $p=\mathrm{NS})$. However, entrainment was completely abolished, and the PSTH showed a large variability in onset latencies of PND (Fig. 5Aii). Nevertheless, despite disruption of entrainment, somatic afferent stimulation increased mean PND frequency during stimulation $(0.40 \pm 0.03$ vs $0.51 \pm 0.02$, control vs stimulus; $p<0.05)$ (Fig. $5 B$ ). Entrainment of respiratory rhythm was restored after washout of isoguvacine. This was confirmed by restoration of the constant latency respiratory pattern at $(372 \pm 43 \mathrm{~ms})$. In contrast, microinjection of isoguvacine outside the lateral and external medial parabrachial nucleus $(n=4)$, as well as injections into the contralateral parabrachial nucleus $(n=3)$, failed to alter entrainment, onset latency, and contraction-induced increases in respiratory frequency (data not shown).
All parabrachial sites were tested using glutamate microinjection before isoguvacine blockade. In the six sites in which isoguvacine blocked entrainment, glutamate evoked an excitatory respiratory response that was typically observed by increases in respiratory frequency. In contrast, in the sites in which isoguvacine failed to prevent entrainment $(n=4)$, glutamate produced no notable respiratory effect. The region of the pons that received microinjections and an example of the location of a single injection site are shown in Figure 5, Ci and Cii. The location of these sites (six effective sites clustered in the lateral parabrachial nucleus and four ineffective sites located medially) and their respective respiratory responses to glutamate microinjection were in general agreement with those reported by Chamberlin and Saper (1994). A summary of the microinjection sites in the ipsilateral parabrachial nucleus are illustrated in Figure 5, Ciii and Civ. Note that injection sites that blocked entrainment were centered in close proximity to the ventrolateral pole of the superior cerebellar peduncle in the lateral and medial divisions of the external parabrachial nucleus. These data suggest that entrainment of respiratory rhythms by somatic stimulation requires the neural integrity of the ipsilateral parabrachial nucleus.

\section{Somatic afferent stimulation depresses expiratory duration ( $n=6$ rats)}

Somatic afferent stimulation immediately after the end of inspiration abruptly terminated post-I activity in the CVN (Fig. $6 A)$. The onset of the following PND occurred prematurely (Fig. 6B). This shortened the respiratory cycle length (from 4 to $3.2 \mathrm{~s}$ ) and increased PND frequency (from 0.25 to $0.32 \mathrm{~Hz}$ ). Group data showed that somatic afferent activation increased significantly respiratory frequency by shortening expiration (Fig. $6 \mathrm{C}$ ). The effect was mediated by a significant reduction in post-I duration. These data suggest that somatic afferent stimulation increases breathing frequency by decreasing expiratory time via inhibition of post-I motor activity.

\section{Responses of medullary respiratory neurons to somatic afferent stimulation ( $n=12$ rats)}

The above results indicate that somatic afferent stimulation entrained respiratory rhythm by selectively reducing expiratory duration. Therefore, we restricted our extracellular recordings to expiratory (post-I and E2) neurons. The effects of somatic afferent stimulation on the firing patterns of post-I and E2 neurons are shown in Figure 7, $A$ and $B$. In this experiment, both post-I (small spike height) and E2 (large spike height) neurons were recorded from a single extracellular microelectrode. Activating somatic afferents produced strong excitation of $\mathrm{E} 2$ neuron while simultaneously terminating the burst of the post-I neuron. These 
changes were associated with a reduction in expiratory duration and hence an increase in PND frequency. Group data showed that somatic afferent stimulation reduced the duration of firing in $71 \%$ of post-I neurons (20 of 28 cells) and increased the peak firing rate in $73 \%$ of E2 neurons ( 11 of 15 cells). The duration of post-I neuronal activity was significantly reduced by somatic stimulation (1116 \pm 400 vs $486 \pm 100 \mathrm{~ms}$, prestimulation vs somatic stimulation; $p<0.05$ ) (Fig. 7C). Conversely, the peak discharge frequency of E2 neurons was significantly increased by stimulation $(54 \pm 15$ vs $87 \pm 20 \mathrm{~Hz}$, prestimulation vs somatic stimulation; $p<0.05)$.

\section{Computational modeling results}

A computational model of the pontomedullary respiratory network developed previously (Rybak et al., 2004a,b) has been used in the present study to simulate the effect of somatic afferent stimulation on the generation of respiratory rhythm. Figure $8 \mathrm{~A}$ shows the schematic of the model (see Materials and Methods and figure legend for details). To apply the model for simulation of the effect of somatic afferent stimulation, an additional interneuron population (S) was incorporated in the model, specifically in the rostral pons (conditionally in the area of the lateral parabrachial nucleus). This population received excitatory input from somatic afferents and provided excitatory input to the medullary E2 population. The weights of excitatory synaptic inputs to the neurons of $S$ population and from $S$ to $E 2$ population were set so that the applied stimuli could provide a moderate level of firing in $S$ neurons $(\sim 40-60$ spikes per second on average), and the level of activation of E2 population was sufficient to completely inhibit activity of the post-I population. We found that both the $1: 1$ entrainment of the respiratory rhythm and the corresponding changes in the firing behavior of E2 and post-I neurons observed in our experiments could be reproduced by the model (Fig. $8 B$ ), with the assumption that the pontine $S$ population directly projected to the E2 population, the activation of which inhibited the post-I population.

According to our suggestion (and our results from the simulations), the somatic afferents provide (via the pontine $S$ population) a strong premature activation of E2 neurons, which inhibits the post-I population (Fig. $8 C, D$, see E2 and post-I traces) and hence initiates the "natural" expiratory off-switching mechanism consisting
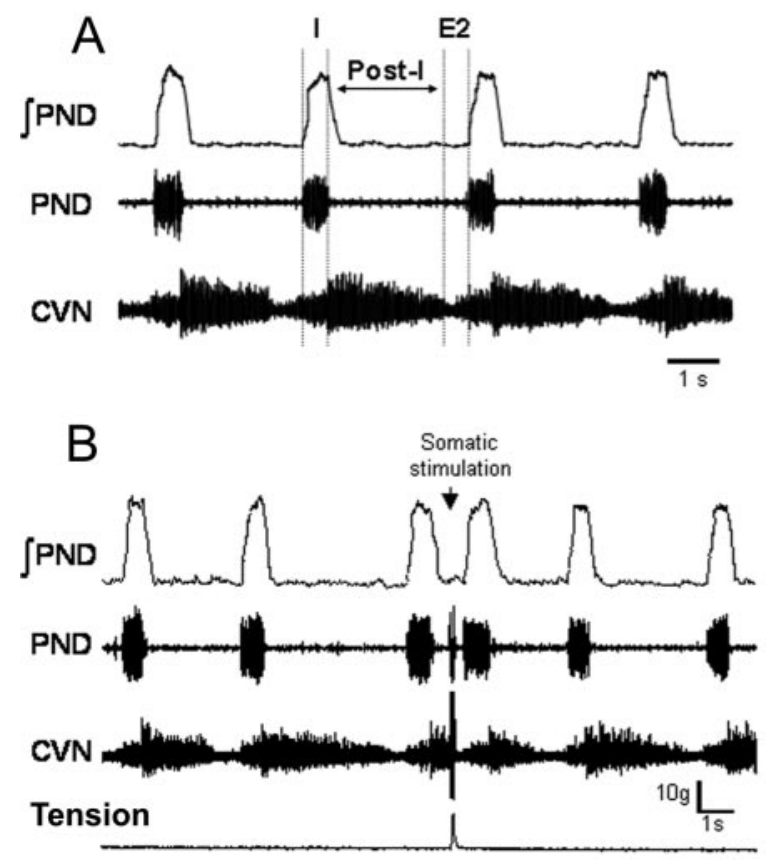

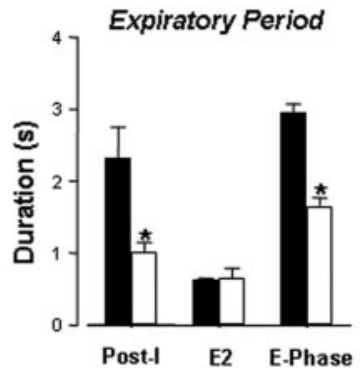

Figure 6. Effect of somatic stimulation on expiratory duration. $\boldsymbol{A}$, Recordings from CVN and PND used to define phases of the respiratory rhythm: I phase, post-I phase, and E2 phase. See explanations in Materials and Methods. $\boldsymbol{B}$, Stimulus-evoked muscle contraction abruptly terminated CVN activity and triggered premature PND bursting. C, Summary data showing effect of somatic stimulation on the duration of I, PI, and E2 phases of the respiratory cycle ( $n=6$ rats). Somatic-evoked increases in respiratory frequency produced a significant reduction in PI duration without altering the duration of I and E2 phases.
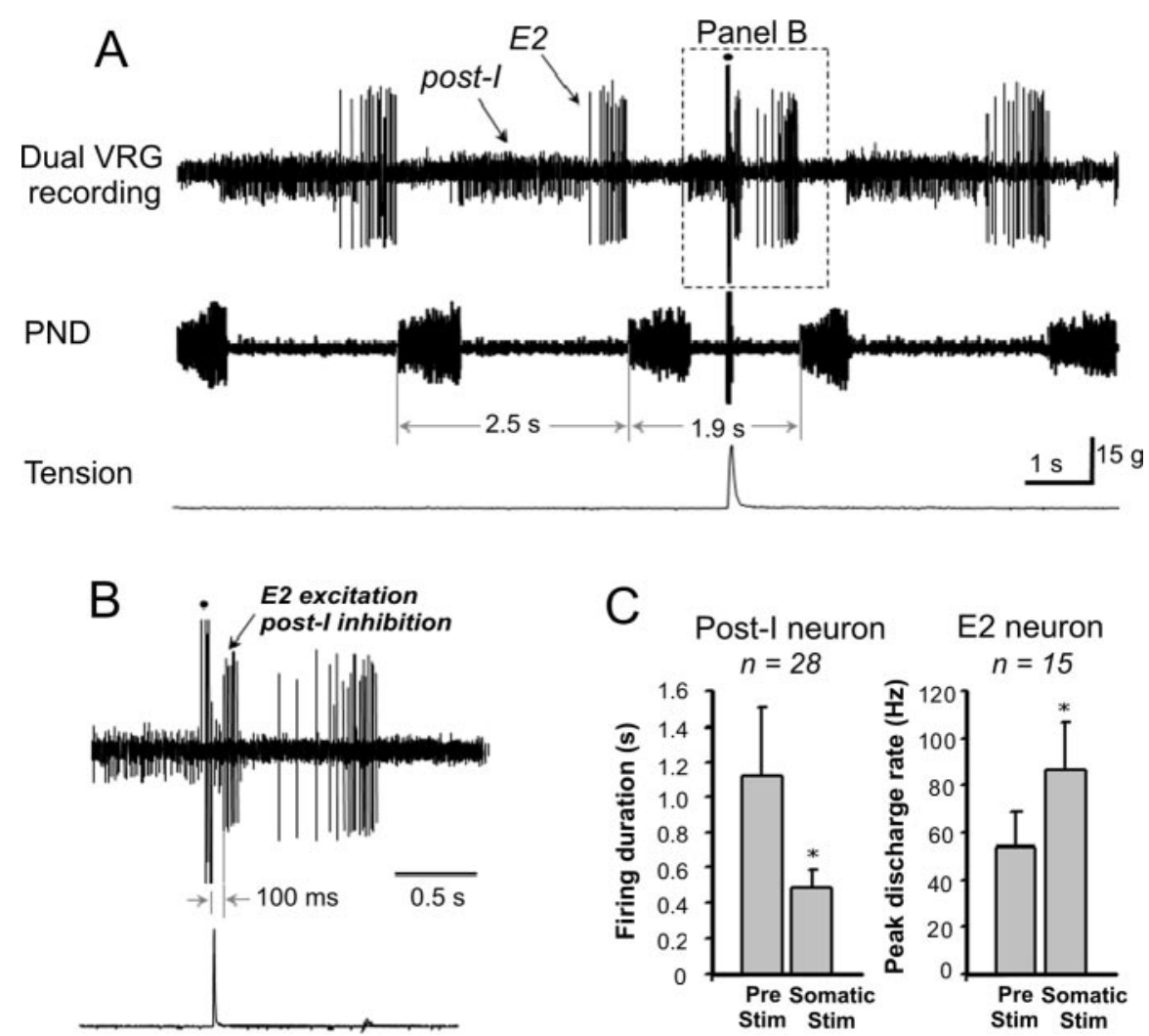

Figure 7. Extracellular recording showing the effect of somatic stimulation on the activity of expiratory (post-I and E2) neurons in the VRG complex. $\boldsymbol{A}$, Simultaneous recording of post-I and $\mathrm{E} 2$ neurons. Somatic stimulation reduced respiratory period duration ( 2.5 vs $1.9 \mathrm{~s}$ ) and altered post-I and E2 neuron activity. $\boldsymbol{B}$, Stimulation abruptly reduced the duration of post-I discharge and increased E2 discharge rate. The dot represents a stimulus artifact. $\boldsymbol{C}$, Summary data showing the effects of somatic stimulation on post-I and E2 neuron activity. ${ }^{*} p<0.05$, significantly different from prestimulation. 

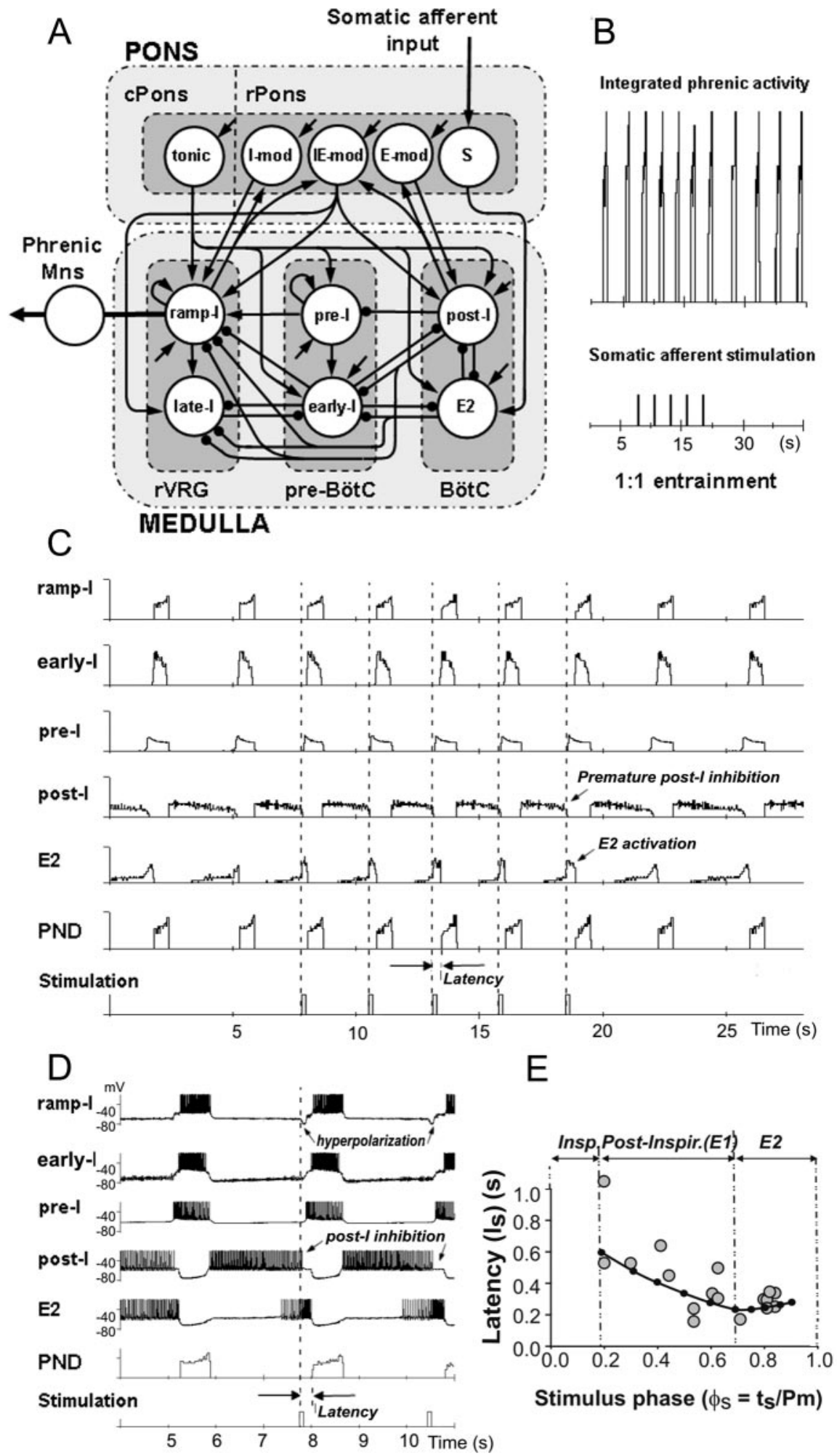

Stimulus phase $\left(\phi_{\mathrm{S}}=\mathrm{t}_{\mathrm{S}} / \mathrm{Pm}\right)$

Figure 8. Modeling the effects of respiratory rhythm entrainment by somatic afferent activation. $A$, Schematic of the model of the pontomedullary respiratory network (modified from Rybak et al., 2004a,b). The circles represent populations of different respiratory neurons. Excitatory and inhibitory synaptic connections are shown by arrows and small filled circles, respectively. For details, see the description in Materials and Methods. Input from somatic afferents provided excitation to the pontine population $\boldsymbol{S}$, which in turn transmitted this excitation to the medullary population of $\mathrm{E} 2$ neurons. $\boldsymbol{B}$, Entrainment $(1: 1)$ of the respiratory rhythm in the model produced by a series of somatic stimuli (bottom trace). $\boldsymbol{C}$, Model performance. Activity of some neural populations (ramp-I, early-I, pre-I, post-I, and E2) and PND is represented by the average histogram of firing frequency (number of spikes per second per neuron; bin of $30 \mathrm{~ms}$ ) in the population. Each modeled population consisted of 50 neurons. The bottom trace shows a series of somatic stimuli. Note that each stimulus produces the following: (1) a strong activation of E2 population, (2) consequent premature inhibition of the post-I population, and (3) early onset of inspiratory burst with respect to the applied

of termination of expiration and onset of inspiration. The advanced onset of inspiration, therefore, is possible if the stimulation is delivered during expiration when the post-I population is active. It is important to note that, because of random distribution of model parameters, the firing behavior of postinspiratory neurons in the model was heterogeneous. A subpopulation of post-I neurons continued firing throughout the expiratory period [neurons of so-called E-dec (expiratory with a decrementing firing pattern) type] until they become completely inhibited by the increasing activity of E2 neurons (Fig. $8 C, D$, post-I traces). Therefore, under normal conditions, the firing activity of post-I and E2 populations overlap and could be defined from the activity of E2 neurons. This distribution of the duration of post-I activity in the model contributed to shaping the incrementing patterns of $\mathrm{E} 2$ neurons. Also, the presence of a subpopulation of post-I neurons of E-dec type was necessary in the model to maintain inhibition of inspiratory and preinspiratory neurons until activity of E2 neurons increased enough to solely maintain inhibition of inspiratory neurons. Therefore, the necessary presence of post-I neurons of E-dec type with firing activity throughout the expiration was considered to be one of modeling predictions. We suggest that the activity of such post-I subpopulation might not be reflected in CVN activity because of a relatively small fraction of these neurons in general or the relatively small fraction of such neurons projecting to CVN. Nevertheless, in our simulations, the activity of all post-I neurons (including those of the E-dec type) were inhibited

$\leftarrow$

stimulus (latency). D, A fragment of same simulation, but each neuron trace shows the membrane potential trajectory of a randomly selected single neuron from each population (ramp-I, early-I, pre-I, post-I, and E2). It is seen that each stimulus produces the following: (1) an increase of firing frequency of the E2 neuron, (2) consequent premature inhibition of the post-I neuron, and (3) early onset of inspiratory burst with latency of $\sim 300 \mathrm{~ms}$. Note also that each stimulus produces a short hyperpolarization of the ramp-I neuron. $\boldsymbol{E}$, The results of modeling investigation for the latency of evoked PND as a function of phase of stimulus application. The black dots connected with the black curve represent the results obtained from the model simulation. The small gray circles represent the results of experimental studies from Figure $4 C$, which have been included for comparison. The vertical dash-dot lines separate inspiratory (Insp), postinspiratory (Post-Inspir.), and E2 phases, as defined in our experimental studies (from Fig. 4C). Note that the latency measured in modeling studies decreases with an increase of stimulus phase during the post-I phase and increases slightly during the E2 phase. 
by the applied stimuli through activation of the E2 population (Fig. 8C,D).

Similar to our experimental findings, the applied stimuli in the model could entrain the respiratory rhythm (compare Fig. $8 \mathrm{~B}$ with Fig. $2 A$ ) by providing activation of the E2 neurons and the resultant inhibition of the post-I neurons (compare Fig. $8 C, D$ with Fig. $7 A, B$ ). The inhibition of the post-I population resulted in a release of pre-I population (in the pre-BötC) from inhibition (Fig. 8C,D). The pre-I neurons then fired, providing excitation to the ramp-I and early-I populations. Finally, the early-I population fired, providing inhibition to E2 population (and maintaining hyperpolarization of the post-I population) to terminate expiration. In turn, the ramp-I population (receiving excitation from the pre-I population and being disinhibited by E2 population) was activated to begin a new inspiratory cycle. The duration and amplitude of the evoked phrenic bursts in the model were the same as the characteristics of the spontaneous phrenic bursts because stimulation during expiration did not affect neural interactions during inspiration.

Similar to our experimental studies, the model demonstrated a temporal delay ( $\sim 300 \mathrm{~ms}$ ) between the stimuli and the evoked PN bursts (latency of evoked inspiration) during repetitive entrainment (compare Fig. $8 C, D$ with Fig. 2C). To further investigate the dependence of latency of the evoked PN bursts on the phase of stimulus application, we performed additional simulations to deliver brief stimuli at different times of expiration. The results are shown in Figure $8 \mathrm{E}$. As seen in this figure, the latency of the evoked PN bursts decreased during the first two-thirds of expiration (approximately corresponding to the experimentally defined post-I phase) and increased slightly during the last onethird of expiration (corresponding to E2 phase). These results closely correlated with our experimental studies (Fig. 4C). Analysis of our model has shown that the monotonic decrease in latency during the first two-thirds of expiration resulted from the characteristic decrementing discharge pattern of post-I neurons, which provide progressively less inhibition to the pre-I population. Therefore, if post-I activity was inhibited (by stimulusevoked excitation of the E2 population) later in expiration, the pre-I neurons would require less time to release from post-I inhibition to initiate the onset of inspiration. The slight increase in latency during the E2 phase resulted from the stimulus-evoked hyperpolarization of ramp-I neurons by the E2 population, which progressively delayed activation of the ramp-I population. The latter mechanism becomes stronger and overcomes the former mechanism by the end of expiration. We suggest that, together, these mechanisms provide an explanation for the reported changes in latency of the evoked PND bursts during post-I and E2 phases (Fig. 4C).

\section{Intracellular recording of medullary post-I and ramp-I neurons ( $n=4$ rats)}

To further investigate the effect of somatic afferent stimulation on medullary respiratory neurons, we used intracellular recordings from post-I and ramp-I neurons in the VRG complex. Three post-I neurons were recorded intracellularly. These neurons exhibited frequency-decrementing firing activity starting from the end of phrenic burst. The membrane potentials of these neurons were $-58,-60$, and $-57 \mathrm{mV}$, respectively (measured during inspiration). Under control conditions (before stimulation was applied), two post-I neurons demonstrated firing patterns throughout the first two-thirds of expiration (Fig. 9A). The third neuron fired throughout the entire expiratory period (Fig. 9B). This finding provides additional evidence that the population of post-I neurons is heterogeneous and that some post-I neurons are indeed active throughout expiration, as suggested in our modeling studies. The first two post-I neurons were tested by application of somatic stimulation. The activity of these neurons was always terminated by the applied stimuli (Fig. 9A). The prestimulus activity pattern duration of $920 \pm 150 \mathrm{~ms}$ was reduced by applied stimulation to $430 \pm 100 \mathrm{~ms}(p<0.05)$. These data confirm our conclusion based on extracellular recordings (Fig. 7) that somatic stimulation indeed inhibits the firing activity of post-I neurons.

The ramp-I neurons recorded intracellularly $(n=8)$ exhibited frequency-incrementing firing during inspiration (defined by PN burst) starting at the onset of PN bursts (Fig. 9C). These neurons had a mean membrane potential of $-57.9 \pm 3.3 \mathrm{mV}$ (measured during expiration). Somatic stimulation entrained the firing bursts in ramp-I neurons in a manner similar to entrainment of PN discharge. In addition, as predicted by the model, each somatic input evoked a membrane hyperpolarization in each intracellularly recorded ramp-I neuron (Fig. 9Ci). The onset latency of these IPSPs was $59.5 \pm 16.3 \mathrm{~ms}$, time-to-peak amplitude was $90.7 \pm 29.3 \mathrm{~ms}$, peak IPSP amplitude was $-7.3 \pm 1.8$ $\mathrm{mV}$, and its duration was $138 \pm 24 \mathrm{~ms}$. Injection of hyperpolarizing current reversed membrane hyperpolarization at potentials close to the calculated $\mathrm{Cl}^{-}$equilibrium potential (Fig. 9Cii), suggesting that somatic input evoked IPSPs in these ramp-I neurons. The stimulus-evoked changes in the compound IPSPs were qualitatively similar to those produced by the computational model (Fig. 8D).

In one recorded ramp-I neuron, the IPSP produced by each stimuli was followed by a single rebound spike (Fig. 9D,Di), as shown to be present in some ramp-I neurons in vivo (Pierrefiche et al., 1999) and likely to be attributable to a transient low-voltage activating calcium current (Rybak et al., 1997a). The peak amplitude of the IPSP was $-10.9 \mathrm{mV}$, and its duration was $52.3 \mathrm{~ms}$ (Fig. 9Dii). The deeper hyperpolarization and faster release from inhibition seen in this neuron likely explains the presence of the rebound spike (Rybak et al., 1997a).

\section{Denervation control experiments ( $n=4$ rats)}

Denervation of somatic afferents was performed to ensure that sensory input producing the entrainment of respiratory rhythms originates in the forelimb. Before transection of the brachial plexus, respiratory rhythms were entrained (1:1) by activation of somatic afferents. After complete transection of the brachial plexus, entrainment and tachypnea evoked by somatic stimulation were completely abolished (data not shown). These findings demonstrate that the respiratory entrainment was mediated by activation of somatic afferent fibers carried in the brachial plexus.

\section{Discussion}

This study supports the existence of a pathway between somatic afferent fibers, pontomedullary respiratory circuits, and phrenic motoneurons that appears to be involved in respiratory-locomotor coupling in mammals. We reported that repetitive somatic afferent stimulation produced an advanced onset of the subsequent respiratory cycle (i.e., inspiration). This entrainment effect of somatic afferent activation was phase dependent, with the largest phase shift occurring when the stimulation was delivered during the postinspiratory phase. Although the phase dependence and entraining effects by somatic afferents has been demonstrated previously in cats, dogs, and geese (Howard et al., 1969; Iscoe and Polosa, 1976; Funk et al., 1992, 1993), this is the first study to show the phase dependence on entrainment and the essential mediating role of the parabrachial nucleus in the reset- 
A
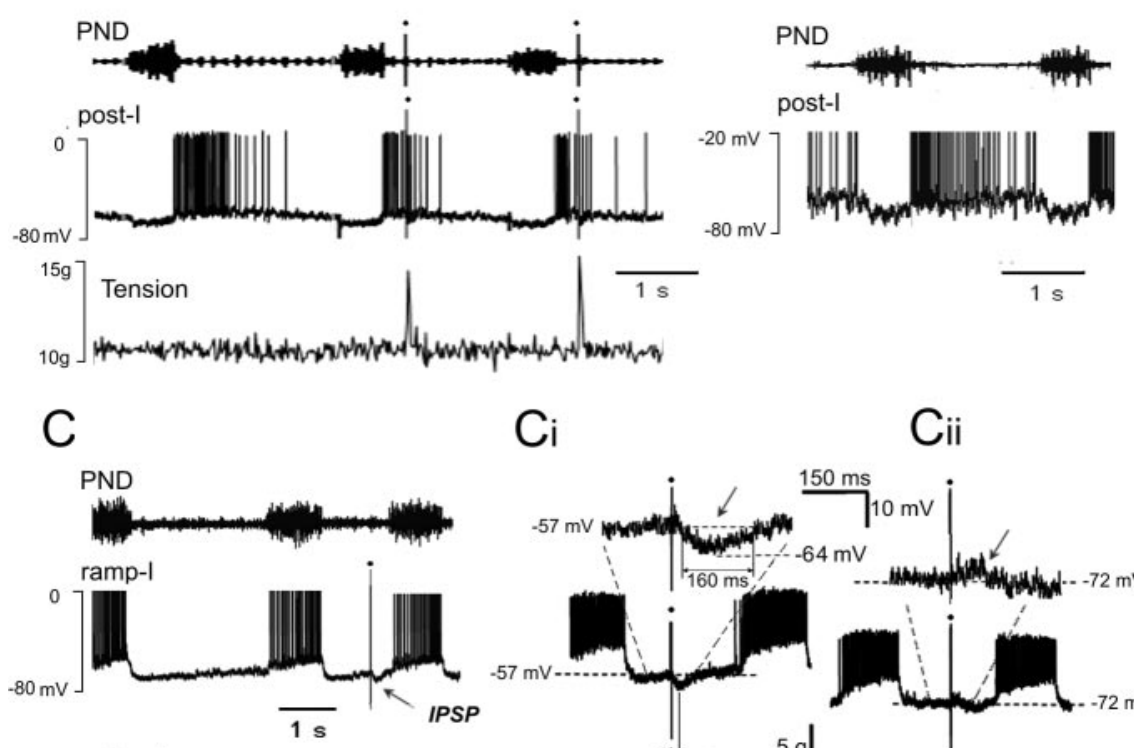

$5 \mathrm{~g}$ Tension

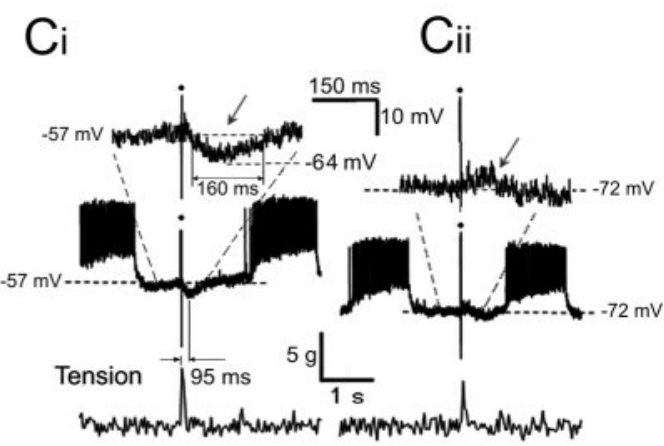

D

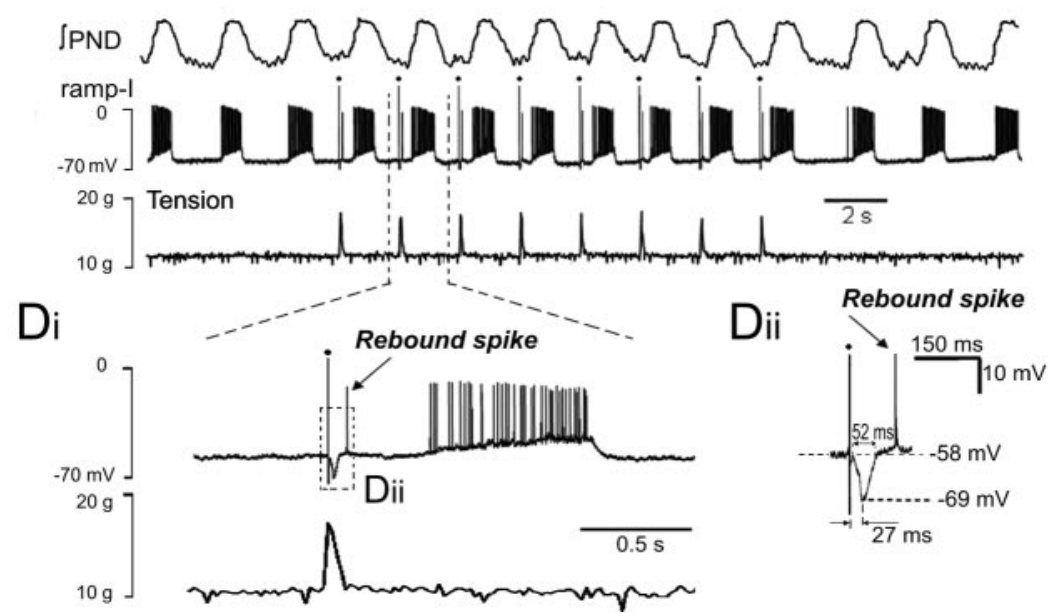

Figure 9. Intracellular recording from identified medullary post-I and ramp-I neurons. $A$, Inhibition of post-I neurons by somatic stimulation. $\boldsymbol{B}$, An example of a post-I neuron with firing activity throughout the entire expiratory period. $\boldsymbol{C}$, Effect of somatic stimulation on a ramp-I neuron. Note a short hyperpolarization (IPSP) produced by the somatic stimulus as predicted by the model (see Fig. 8D). Ci, The same neuron. The IPSP (pointed by a small arrow) was produced by each somatic stimulus. Cii, The evoked IPSP was reversed by injection of hyperpolarizing current. D-Dii, Another ramp-I neuron. Somatic stimulation entrained PND and produced IPSPs in this neuron that were followed by rebound spikes. Note that this neuron had deeper IPSPs than the neuron shown in $\mathbf{C}$, and there was a faster release of this cell from inhibition.

ting of respiratory rhythm by somatic afferent input because chemical inactivation of this region eliminated entrainment.

\section{Phase resetting of respiratory rhythms}

One-to-one phase coupling of the respiratory network by somatic afferent input has been reported in many mammalian species (Iscoe and Polosa, 1976; Bramble and Carrier, 1983; Funk et al., 1992). However, as we found in the rat, mammals demonstrate varying degrees of entrainment. In addition to $1: 1$ phase coupling, multiple coupling patterns have been reported (Iscoe, 1981; Bramble and Carrier, 1983; Funk et al., 1992; Ainsworth et al., 1996). The fact that 1:1 phase coupling was produced over a range of contraction frequencies in this and previous studies suggests that the afferent-evoked influence on the pontomedullary circuit involves a complete resetting of the respiratory rhythm generator. Moreover, based on our experimental and modeling studies, we suggest that input from somatic afferents provides an advance activation of the "natural" expiratory off-switching mechanism present in the pontomedullary respiratory circuit.

We also found that phase resetting was strongly dependent on the timing of somatosensory input within the respiratory cycle. We showed that, in agreement with previous studies, the most effective phase for phase switching was expiration (St. John and Zhou, 1989, 1991). This phase dependence was also observed in contractionevoked inhibition of post-I neurons and contraction-evoked IPSPs in intracellularly recorded ramp-I neurons. The entrainment effect of stimuli delivered during inspiration was inconsistent and requires additional investigation. Together, these data suggest that entrainment of respiratory rhythm by somatic afferents is phase dependent and is provided by specific interactions within the pontomedullary respiratory network.

\section{Role of the parabrachial nucleus in respiratory-locomotor coupling}

The parabrachial nucleus is a key structure in central autonomic networks and exerts profound influences on a variety of autonomic and respiratory functions (Chamberlin and Saper, 1992, 1994). Regarding respiratory control, electrical or chemical stimulation of the dorsolateral region of the parabrachial nucleus during expiration produced premature initiation of inspiration and increased amplitude of phrenic nerve discharge (Cohen, 1971; McCrimmon et al., 1986; Miura and Takayama, 1991). This suggests the existence of parabrachial neurons that can contribute to on-switching of inspiration. Chamberlin and Saper (1994) confirmed this possibility and described three distinct sites within the parabrachial nucleus that mediated different respiratory responses, suggesting that the parabrachial nucleus affects breathing in multiple ways and does not function solely as an inspiratory off-switch mechanism, as originally proposed by Lumsden (1923).

We report that the pontine regions responsible for mediating entrainment were centered exclusively in the ipsilateral dorsolateral region of the parabrachial nucleus around the ventrolateral border of the superior cerebellar peduncle and the external lateral subnucleus (Fig. 5Ci-Civ). When we microinjected glutamate into these sites, the expiratory interval was shortened and the subsequent phrenic burst occurred prematurely. These regions are similar to those reported by Chamberlin and Saper (1994), 
which produced hyperpnea after glutamate stimulation, and consistent with sites involved in inspiratory on-switching. However, our data also suggests a potential role for spinal somatosensory pathways in activating the inspiratory on-switching neurons in this region. Support for this comes from the direct projection of dorsal horn neurons to the ipsilateral dorsolateral parabrachial nucleus (Cechetto et al., 1985; Bernard et al., 1995; Feil and Herbert, 1995).

It should be noted that, despite blockade of entrainment, inactivating the ipsilateral dorsolateral parabrachial nucleus failed to affect the overall evoked increase in respiratory frequency during somatic afferent stimulation (Fig. 5B). These findings lead us to propose that somatic stimulation modulates respiratory activity by at least two independent neural routes: (1) a spinopontomedullary pathway controlling the phase of inspiration relative to an afferent stimulus; and (2) a neural circuit that mediates tachypnea. The latter pathway may involve other medullary neurons possibly through affecting an excitatory tonic drive to the respiratory circuitry. Nevertheless, our data failed to support a singular role for this parabrachial region in mediating the tachypnea response to somatic stimulation.

\section{Responses of medullary respiratory neurons to somatic stimulation}

Extracellular recording of medullary respiratory neurons exhibited consistent responses of E2 and post-I neurons to somatic afferent stimulation. Somatic stimulation evoked excitatory effects on the activity of E2 neurons and reduced the duration of firing of post-I neurons (Fig. 7). These experimental data, however, did not allow us to determine whether the somatic stimulation initially activated E2 neurons, which then inhibited post-I neurons, or whether it first inhibited post-I neurons, which in turn disinhibited E2 neurons. However, our modeling study provided support to the former mechanism. Moreover, the model predicted that E2 neurons (initially activated by afferent stimulation) should also produce a transient membrane hyperpolarization in ramp-I neurons (Fig. $8 D$ ). This prediction was confirmed experimentally (Fig. 9C,D). Although we do not feel that somatic sensory-evoked IPSPs in ramp-I neurons contribute significantly to the central phase-switching mechanisms, our modeling studies suggest that these IPSPs may be responsible for the increased latency of the evoked inspiratory bursts during late expiration. Nevertheless, these experimental findings provide additional validation of both the applied model for the respiratory network and the suggested neural mechanism for entrainment.

\section{Putative synaptic mechanisms: insights from modeling studies}

In this study, we used the model of pontomedullary network that had been developed previously without the account of the experimental results described. We have only used the assumption that activation of somatic afferents provides input to the medullary E2 neurons via an excitatory projection from the rostral pons. According to the model, the post-I population is active during the early portion of expiration and inhibits all other respiratory neural populations in the medulla (Fig. $8 A, C, D$ ). This is supported by previously published data (Lindsey et al., 1987; Segers et al., 1987; Ezure, 1990; Bianchi et al., 1995; Okazaki et al., 2002; Shen and Duffin, 2002). As a result of frequency adaptation, the activity of post-I neurons is progressively reduced throughout expiration, thus decreasing post-I inhibition of the E2 neural population with time. In the middle of expiration, the E2 population is activated and provides feedback inhibition to the post-I popula- tion, which first reduces and finally terminates post-I activity (Lindsey et al., 1987; Segers et al., 1987; Ezure, 1990; Jiang and Lipski, 1992; Bianchi et al., 1995; Duffin et al., 2000; Ezure et al., 2003). In our model, inhibition of the post-I population disinhibits the pre-I population in the pre-BötC, which fires and initiates switching to inspiration (Richter, 1996) by activation of early-I and ramp-I populations. The early-I population finally inhibits all expiratory populations (E2 and post-I) during inspiration (Segers et al., 1987; Ezure, 1990; Bianchi et al., 1995). This suggested mechanism also provides an explanation for why early expiration is the most sensitive phase for entrainment of the respiratory rhythm produced by activation of somatic afferents, as well as why latency of the evoked phrenic bursts decrease during the initial portion of expiration and increase at the end of expiration (see the description above in Results, Computational modeling results).

The model could realistically reproduce a number of experimental phenomena including the following: (1) entrainment of respiratory rhythm; (2) activation of the E2 neurons; (3) inhibition of the post-I neurons; (4) a $300 \mathrm{~ms}$ latency in the onset of evoked phrenic burst during entrainment by repetitive stimulation; and (5) phase dependencies of both the effect of somatic afferent stimulation and the latency of the evoked phrenic burst. Moreover, the model predicted a heterogeneity of firing burst durations of postinspiratory neurons and the presence of short hyperpolarization of ramp-I neurons, which was confirmed experimentally (Fig. 9). Therefore, we believe that our model may realistically reproduce the cellular mechanism by which the somatic afferents entrain the respiratory rhythm. Based on our model, we suggest that somatic afferent input evokes (via intermediate pontine neurons) excitation of medullary augmenting expiratory (E2) neurons, which in turn inhibit postinspiratory neurons and hence shorten the duration of expiration. At the same time, the small number of intracellular recording of post-I neurons and the lack of intracellular recording of medullary pre-I and E2 neurons in the present study do not allow us to fully validate the neural mechanism of entrainment and more broadly the expiratory off-switch mechanism suggested by the model. Systematic intracellular recordings from medullary pre-I, E2, and post-I neurons are necessary for additional investigation of the suggested neural mechanisms.

\section{Physiological significance of respiratory coupling during locomotion}

The issue about which type of somatic afferents was activated in the present study requires additional investigation. Although the idea was that the somatic stimulus used in this study would primarily activate muscle afferents, we cannot rule out the possibility that activation of other afferent populations (i.e., mechanical, chemical, low-threshold vs high-threshold, and nociceptor vs non-nociceptor) may have contributed to somatic afferent activation either directly or indirectly by the local release of neurostimulatory substances (i.e., $\mathrm{K}^{+}, \mathrm{H}^{+}$, adenosine, etc.) from contracting skeletal muscle. However, it should be noted that previous studies have clearly demonstrated that selective activation of large myelinated primary muscle spindles was ineffective as a respiratory stimulus (Hodges and Mathews, 1968; Waldrop et al., 1984).

Functionally, the coupling of respiratory and locomotor systems may increase the efficiency of locomotion by allowing locomotor musculature to contribute to the production of airflow, thereby reducing the metabolic cost of breathing (Bramble and Carrier, 1983; Funk et al., 1997). In addition, the phase timing of 
respiration is a particularly important issue during locomotion in quadrupeds that must contend with mechanical interference between the opposing actions of the diaphragm and visceral contents of the abdomen (Bramble and Carrier, 1983). Furthermore, the fact that respiratory and locomotor rhythm patterns occur over a range of coupled frequencies during bipedal gait suggests that the fundamental circuitry and neural connectivity is conserved in humans.

In conclusion, we have shown that sensory input from somatic afferents rapidly and robustly entrains breathing by modulating the activity of medullary respiratory neurons via descending connections from the lateral parabrachial nucleus. We also concluded that medullary augmenting expiratory neurons play a pivotal role in somatic afferent-evoked entrainment of the respiratory rhythm. It appears that advanced activation of these neurons by somatic afferent input provides early initiation of the natural inspiratory on-switching mechanism operating in the medulla.

\section{References}

Ainsworth DM, Smith CA, Henderson KS, Dempsey JA (1996) Breathing during exercise in dogs—passive or active? J Appl Physiol 81:586-595.

Bernard JF, Dallel R, Raboisson P, Villanueva L, Le Bars D (1995) Organization of the efferent projections from the spinal cervical enlargement to the parabrachial area and periaqueductal gray: a PHA-L study in the rat. J Comp Neurol 353:480-505.

Bianchi AL, Denavit-Saubie M, Champagnat J (1995) Central control of breathing in mammals: neuronal circuitry, membrane properties and neurotransmitters. Physiol Rev 75:1-45.

Boscan P, Paton JFR (2002) Nociceptive afferents selectively modulate the cardiac component of the peripheral chemoreceptor reflex via actions within the solitary tract nucleus. Neuroscience 110:319-328.

Bramble DM, Carrier DR (1983) Running and breathing in mammals. Science 219:251-256.

Brice AG, Forster HV, Pan LG, Funahashi A, Hoffman MD, Murphy CL, Lowry TF (1988) Is the hyperpnea of muscular contractions critically dependent on spinal afferents? J Appl Physiol 64:226-233.

Brown DR, Forster HV, Pan LG, Brice AG, Murphy CL, Lowry TF, Gutting SM, Funahashi A, Hoffman M, Power S (1990) Ventilatory response of spinal cord-lesioned subjects to electrically induced exercise. J Appl Physiol 68:2312-2321.

Cechetto DF, Standaert DG, Saper CB (1985) Spinal and trigeminal dorsal horn projections to the parabrachial nucleus in the rat. J Comp Neurol 240:153-160.

Chamberlin NL, Saper CB (1992) Topographical organization of cardiovascular responses to electrical and glutamate microstimulation of the parabrachial nucleus in the rat. J Comp Neurol 326:245-262.

Chamberlin NL, Saper CB (1994) Topographical organization of respiratory responses to glutamate microstimulation of the parabrachial nucleus in the rat. J Neurosci 4:6500-6510.

Cohen M (1971) Switching of the respiratory phases and evoked phrenic responses produced by rostral pontine electrical stimulation. J Physiol (Lond) 217:133-158.

Cohen MI (1979) Neurogenesis of respiratory rhythm in the mammal. Physiol Rev 59:1105-1173.

Cohen MI, Feldman JL (1977) Models of respiratory phase switching. Fed Proc 36:2367-2374.

Dejours P (1967) Neurogenic factors in the control of ventilation during exercise. Circ Res 20-21 [Suppl 1]:I146-I153.

Dick TE, Bellingham MC, Richter DW (1994) Pontine respiratory neurons in anesthetized rats. Brain Res 636:259-269.

Dubayle D, Viala D (1996) Localization of the spinal respiratory rhythm generator by an in vitro electrophysiological approach. NeuroReport 7:1175-1180.

Dubayle D, Viala D (1998) Entrainment of the medullary respiratory generators by electrical stimulation in the cervical grey matter on in vitro preparations of newborn rats. Neurosci Lett 248:204-208.

Duffin J, Tian G, Peever J (2000) Functional synaptic connections among respiratory neurons. Respir Physiol 122:237-246.

Eldridge FL, Millhorn DE, Waldrop TG (1981) Exercise hyperpnea and lo- comotion: parallel activation from the hypothalamus. Science 211:844-846.

Elsen FP, Ramirez J (1998) Calcium currents of rhythmic neurons recorded in the isolated respiratory network of neonatal mice. J Neurosci $18: 10652-10662$.

Ezure K (1990) Synaptic connections between medullary respiratory neurons and consideration on the genesis of respiratory rhythm. Prog Neurobiol 35:429-450.

Ezure K, Tanaka I, Saito Y (2003) Brainstem and spinal projections of augmenting expiratory neurons in the rat. Neurosci Res 45:41-51.

Feil K, Herbert H (1995) Topographical organization of spinal and trigeminal somatosensory pathways to the rat parabrachial and Kolliker-fuse nuclei. J Comp Neurol 353:506-528.

Feldman JL (1986) Neurophysiology of breathing in mammals. In: Handbook of physiology, Sec 1, The nervous system, Vol IV, Intrinsic regulation system of the brain (Bloom FE, ed), pp 463-524. Bethesda, MD: American Physiological Society.

Fernandes A, Galbo H, Kjer M, Mitchell M, Secker NH, Thomas S (1990) Cardiovascular and ventilatory responses to dynamic exercise during epidural anaesthesia in man. J Physiol (Lond) 420:281-293.

Frermann D, Keller BU, Richter DW (1999) Calcium oscillations in rhythmically active respiratory neurons in the brainstem of the mouse. J Physiol (Lond) 515:119-131.

Funk GD, Milsom WK, Steeves JD (1992) Coordination of wingbeat and respiration in the Canada goose. I. Passive wing flapping. J Appl Physiol 73:1014-1024.

Funk GD, Sholomenko GN, Valenzuela IJ, Steeves JD, Milsom WK (1993) Coordination of wing beat and respiration in Canada goose during free flight. J Exp Biol 175:317-323.

Funk GD, Valenzuela IJ, Milsom WK (1997) Energetic consequences of coordinating wing beat and respiration in birds. J Exp Biol 200:915-920.

Gaytan SP, Calero F, Nunez-Abades PA, Morillo AM, Pasaro R (1997) Pontomedullary efferent projections of the ventral respiratory neuronal subsets of the rat. Brain Res Bull 42:323-334.

Hodges HJF, Mathews PBC (1968) The ineffectiveness of excitation of primary endings of the muscle spindle by vibration as a respiratory stimulant in the decerebrate cat. J Physiol (Lond) 194:555-563.

Howard P, Braomberger-Barnea B, Fitzgerald RS, Bane HN (1969) Ventilatory responses to peripheral nerve stimulation at different times in the respiratory cycle. Respir Physiol 7:389-398.

Iscoe S (1981) Respiratory and stepping frequencies in conscious exercising cats. J Appl Physiol 51:835-839.

Iscoe S, Polosa C (1976) Synchronization of respiratory frequency by somatic afferent stimulation. J Appl Physiol 40:138-148.

Jiang C, Lipski J (1992) Synaptic inputs to medullary respiratory neurons from superior laryngeal afferents in the cat. Brain Res 584:197-206.

Johansson JE (1893) Ueber die einwirkung der muskeltaetigkeit auf die atmung und die herztaetigkeit. Scand Arch Physiol 5:20-66.

Kao FF (1963) An experimental study of the pathway involved in exercise hyperpnea employing cross-circulation technique. In: The regulation of human respiration (Cunningham DJC, Lloyd BB, eds), pp 461-502. Oxford: Blackwell.

Kaufman MP (1995) Afferents from limb skeletal muscle. In: Regulation of breathing, Ed 2 (Dempsey JA, Pack AI, eds), pp 583-616. New York: Marcel Dekker.

Krogh A, Lindhard J (1913) The regulation of respiration and circulation during the initial stages of muscular work. J Physiol (Lond) 47:112-136.

Lindsey BG, Segers LS, Shannon R (1987) Functional associations among simultaneously monitored lateral medullary respiratory neurons in the cat. II. Evidence for inhibitory actions of expiratory neurons. J Neurophysiol 57:1101-1117.

Lumsden T (1923) Observations on the respiratory centers in the cat. J Physiol (Lond) 57:153-160.

McCloskey DI, Mitchell JH (1972) Reflex cardiovascular and respiratory responses originating in exercising muscle. J Physiol (Lond) 224:173-186.

McCrimmon DR, Feldman JL, Speck DF, Ellenberger HH, Smith JC (1986) Functional heterogeneity of dorsal, ventral and pontine respiratory groups revealed by micropharmacological techniques. Acta Physiol Scand [Suppl] 259:1-42.

Mitchell JH, Reardon WC, McCloskey DI (1977) Reflex effects on circulation and respiration from contracting skeletal muscle. Am J Physiol 233:H374-H378. 
Miura A, Takayama K (1991) Circulatory and respiratory responses to glutamate stimulation of the lateral parabrachial nucleus of the cat. J Auton Nerv Syst 21:195-202.

Morin D, Viala D (2002) Coordination of locomotor and respiratory rhythms in vitro are critically dependent upon hindlimb sensory inputs. J Neurosci 22:4756-4765.

Ogilvie MD, Gottschalk A, Anders K, Richter DW, Pack AI (1986) A network model of respiratory rhythmogenesis. Am J Physiol 263:R962-R975.

Okazaki M, Takeda R, Yamazaki H, Haji A (2002) Synaptic mechanisms of inspiratory off-switching evoked by pontine pneumotaxic stimulation in cats. Neurosci Res 44:101-110.

Pan LG, Forster HV, Wurster RD, Murphy CL, Brice AG, Lowry TF (1990) Effect of partial spinal cord ablation on exercise hyperpnea in ponies. J Appl Physiol 69:1821-1827.

Paton JFR (1996a) A working heart-brainstem preparation of the mouse. Neurosci Methods 65:63-68.

Paton JFR (1996b) The ventral medullary respiratory network of the mature mouse studied in a working heart-brainstem preparation. J Physiol (Lond) 493:819-831.

Pierrefiche O, Haji A, Bischoff A, Richter DW (1999) Calcium currents in respiratory neurons of the cat in vivo. Pflügers Arch 438:817-826.

Potts JT, Paton JFR (2000) Dorsolateral pons mediates skeletal muscle contraction induced respiratory coupling. Soc Neurosci Abstr 26:546.13.

Potts JT, Paton JFR (2001) Locomotor pacing of respiratory network involves early excitation of augmenting expiratory (E-AUG) neurons. Soc Neurosci Abstr 27:173.4.

Potts JT, Paton JFR (2002) Locomotor pacing of respiratory network involves early excitation of augmenting expiratory (E-AUG) neurons. FASEB J 16:A809.

Potts JT, Spyer KM, Paton JFR (2000) Somatosympathetic reflex in a working heart-brainstem preparation of the rat. Brain Res Bull 53:59-67.

Richter D, Ballantyne D, Remmers JE (1986) How is the respiratory rhythm generated? A model. News Physiol Sci 1:109-112.

Richter DW (1996) Neural regulation of respiration: rhythmogenesis and afferent control. In: Comprehensive human physiology, Vol II (Gregor R, Windhorst U, eds), pp 2079-2095. Berlin: Springer.

Rybak IA, Paton JFR, Schwaber JS (1997a) Modeling neural mechanisms for genesis of respiratory rhythm and pattern. I. Models of respiratory neurons. J Neurophysiol 77:1994-2006.

Rybak IA, Paton JFR, Schwaber JS (1997b) Modeling neural mechanisms for genesis of respiratory rhythm and pattern. II. Network models of central respiratory pattern generator. J Neurophysiol 77:2007-2026.

Rybak IA, Paton JFR, Schwaber JS (1997c) Modeling neural mechanisms for genesis of respiratory rhythm and pattern. III. Comparison of model performance during afferent nerve stimulation. J Neurophysiol 77:2027-2039.

Rybak IA, Ptak K, Shevtsova NA, McCrimmon DR (2003a) Sodium currents in neurons from the rostroventral medulla of the rat. J Neurophysiol 90:1635-1642.

Rybak IA, Shevtsova NA, St. John WM, Paton JFR, Pierrefiche O (2003b) Endogenous rhythm generation in the pre-Bötzinger complex and ionic currents: modelling and in vitro studies. Eur J Neurosci 18:239-257.

Rybak IA, Shevtsova NA, Paton JFR, Dick TE, St. John WM, Mörschelm M, Dutschmann M (2004a) Modeling the ponto-medullary respiratory network. Respir Physiol Neurobiol 143:307-319.

Rybak IA, Shevtsova NA, Paton JFR, Pierrefiche O, St. John WM, Haji A (2004b) Modelling respiratory rhythmogenesis: focus on phase switching mechanisms. Adv Exp Med Biol 551:189-194.

Rybak IA, Shevtsova NA, Ptak K, McCrimmon DR (2004c) Intrinsic bursting activity in the pre-Bötzinger complex: role of persistent sodium and potassium currents. Biol Cybern 90:59-74.

Segers LS, Shannon R, Saporta S, Lindsey BG (1987) Functional associations among simultaneously monitored lateral medullary respiratory neurons in the cat. I. Evidence for excitatory and inhibitory connections of inspiratory neurons. J Neurophysiol 57:1078-1100.

Shen L, Duffin J (2002) Caudal expiratory neurons in the rat. Pflügers Arch 444:405-410.

St. John WM (1998) Neurogenesis of patterns of autonomic ventilatory activity. Prog Neurobiol 56:97-117.

St. John WM, Paton JFR (2000) Characterization of eupnea, apneusis and gasping in a perfused rat preparation. Respir Physiol 123:201-213.

St. John WM, Zhou D (1989) Differing control of neural activities during various portions of expiration in the cat. J Physiol (Lond) 418:189-204.

St. John WM, Zhou D (1991) Rostral pontile mechanisms regulate durations of expiratory phases. J Appl Physiol 71:2133-2137.

St. John WM, Rybak IA, Paton JFR (2002) Switch from eupnea to fictive gasping after blockade of inhibitory transmission and potassium channels. Am J Physiol Regul Integr Comp Physiol 283:R721-R731.

von Euler C (1986) Brain stem mechanisms for generation and control of breathing pattern. In: Handbook of physiology, Sec 3, The respiratory system, Vol II, Control of breathing (Cherniak NS, Widdicombe JG), pp 1-67. Bethesda, MD: American Physiological Society.

Waldrop TG, Rybicki K, Kaufman MP (1984) Chemical activation of group I and II muscle afferents has no cardiorespiratory effects. J Appl Physiol $56: 1223-1228$.

Wang W, Fung M-L, St. John WM (1993) Pontile regulation of ventilatory activity in the adult rat. J Appl Physiol 74:2801-2811.

Zunzt N, Geppert J (1886) Ueber die nature der normalen atemreize und den ort ihrer wirkung. Arch Ges Physiolo 38:337-338. 